\title{
ASSESSING AND DEFINING EXPLICIT PROCESSES IN VISUOMOTOR ADAPTATION
}

By

Sarvenaz Heirani Moghaddam

Supervisor: Erin K. Cressman

Committee: Romeo Chua \& Anthony N. Carlsen

Thesis submitted to the University of Ottawa in partial fulfillment of the requirements for the

MASTERS OF SCIENCE

in Human Kinetics

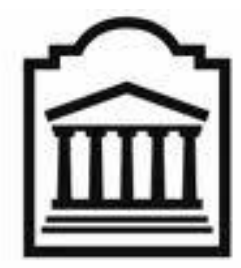

uOttawa

\section{L'Université canadienne}

Canada's university

School of Human Kinetics, Faculty of Health Sciences

University of Ottawa

(C) Sarvenaz Heirani Moghaddam, Ottawa, Canada, 2020 


\section{Running Head: EXPLICIT AND IMPLICIT VISUOMOTOR ADAPTATION}

\section{Statement of Contributors}

I hereby declare that I am the sole author of this Master's of Science thesis. My contributions include, a review of literature in the area of interest, participant recruitment, participant testing, data collection, data compilation, statistical analyses, and the write-up of this thesis document. All of these duties were performed under the guidance and mentorship of my research supervisor, Dr. Erin K. Cressman.

The experiment in this thesis was performed in collaboration with my research supervisor, Dr. Erin K. Cressman, who provided editorial corrections and feedback, and is the co-author of the article presented in this thesis. 


\section{Running Head: EXPLICIT AND IMPLICIT VISUOMOTOR ADAPTATION}

\section{Acknowledgements}

I am grateful for my experience as a master's student in the Sensorimotor Control lab at the University of Ottawa. The past two years have been transformational and awesome. I've learned many skills and have met great people who have added positive values to my life. I am grateful.

Dr. Romeo Chua, I would not have started this master's degree if it wasn't for you and your encouragement. It all started with the KIN330 course taught by you at UBC in 2016. Even though lectures were at 8am, you were joyous and happy to teach us complex concepts in human motor control. I will never forget how you had all of the students' names (even my long name!) memorized after the first lecture. Also, the opportunity to experience research for the first time in your lab gave me confidence to pursue a master's with Dr Erin Cressman who is the kindest and smartest supervisor. I love and thank both of you for every piece of knowledge you have given me and all the shared experiences we have had together.

Dr. Erin Cressman, you are the best mentor I have ever had. I admire everything you do. You are an exceptional professor, amazing researcher and a phenomenon writer. Besides academics, the way you walk through life and your healthy habits have taught me that taking care of myself is the best thing I can do to be able to be a supportive and compassionate daughter, sister,

friend, partner, and a colleague. You are a caring nurturer and mentor who has taught me many skills including patience because just like writing this thesis, life is a marathon. I am a better person because of you. Thank you. 


\section{Running Head: EXPLICIT AND IMPLICIT VISUOMOTOR ADAPTATION}

Dr. Tony Carlsen, thank you for your continuous support, insights and lessons over the past two years. Thank you for great constructive feedback on my writing and various presentations.

My lab mates, my team! You made my time at uOttawa so fun. Besides the research work, you have been the best support system when I needed it most. We have worked, laughed, partied and cried. I could not have asked for a better team of researchers.

Everything I have, anything I can do and the person I am today, is because of my mom's sacrifices. Mom, I love you. Thank you so much for everything you have done for me. Besides literally creating me and being the best mom ever, you have been my rock and the most supportive person through this journey. You are my superhero.

My life partner, Mark, thank you for staying by my side through it all. I appreciate you. You always remind me that everything will be okay when I constantly freak-out over small stuff. I am truly blessed for your ongoing support. I would not have been able to finish this degree without you. 


\section{Table of Contents}

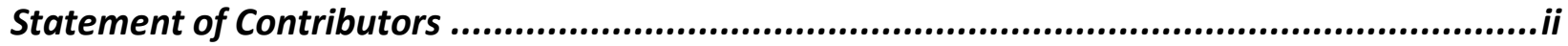

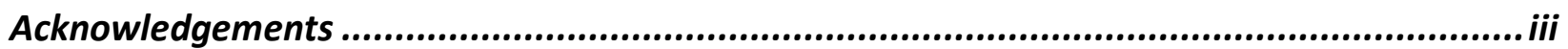

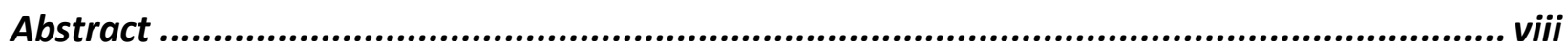

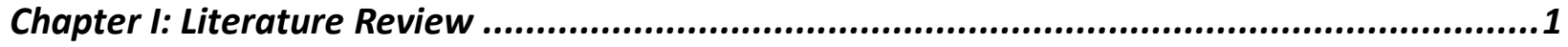

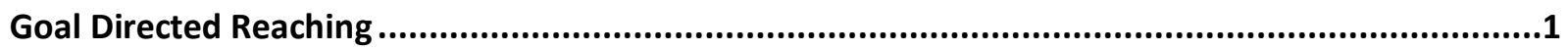

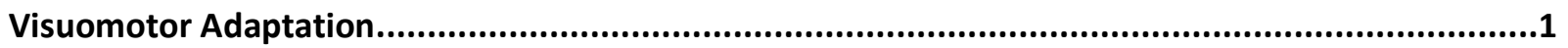

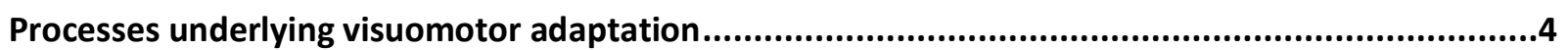

Explicit processes in visuomotor adaptation across methods of assessment ...............................6

Rationale and Current Research Objectives...............................................................................10

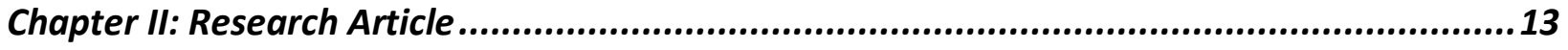

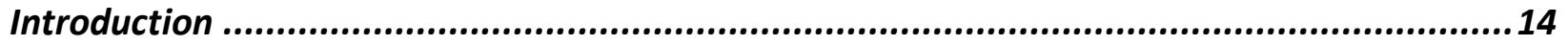

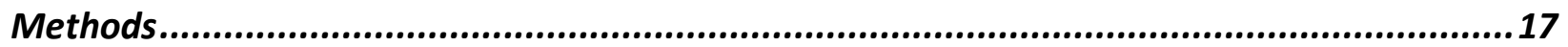

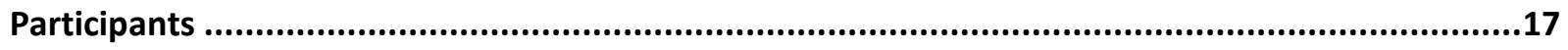

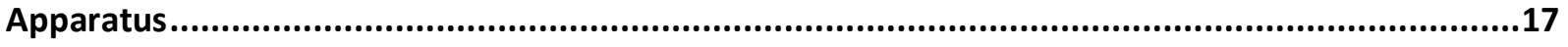

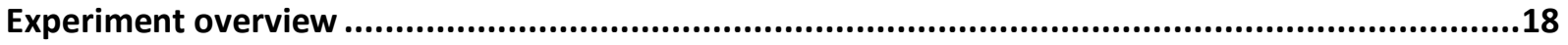

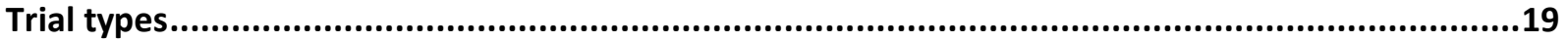

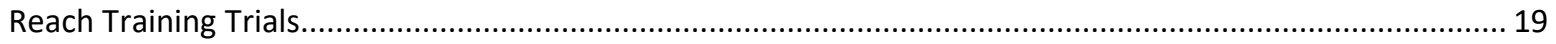

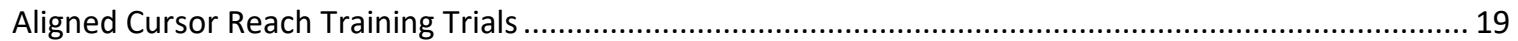

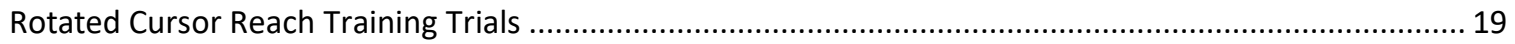

Trials to assess Explicit and Implicit contributions to Visuomotor Adaptation ................................................ 20

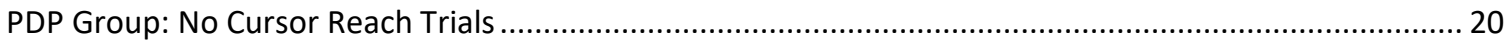

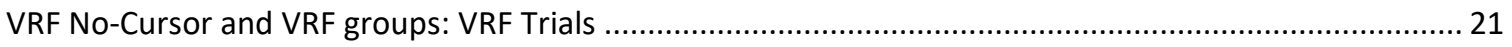

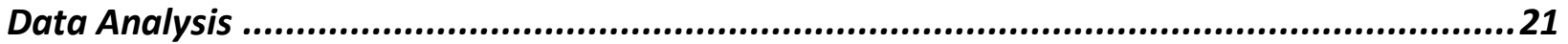

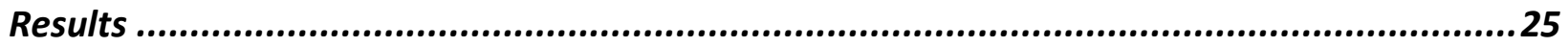

Awareness as assessed via the Post-Experiment Questionnaire (PEQ) and Drawing Task (DT)...................... 25

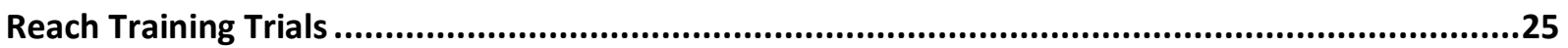

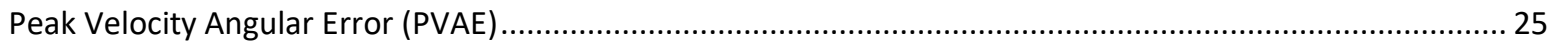

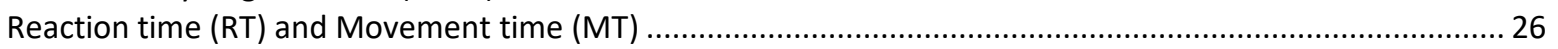

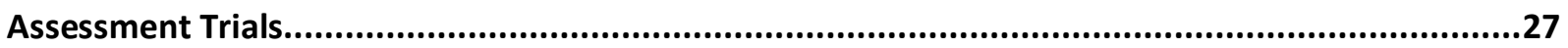

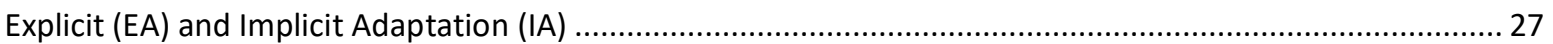

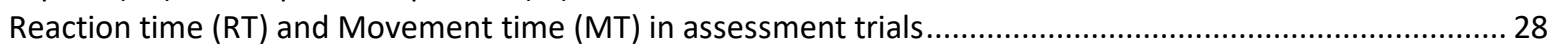

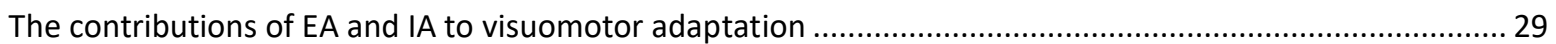

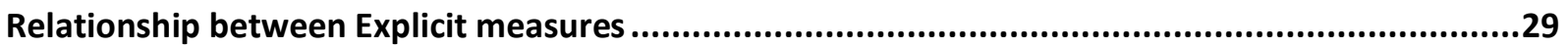

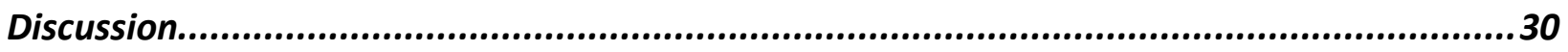

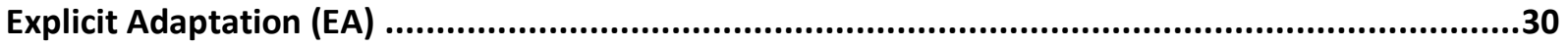

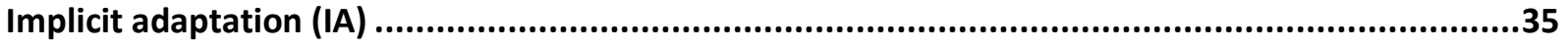


Running Head: EXPLICIT AND IMPLICIT VISUOMOTOR ADAPTATION

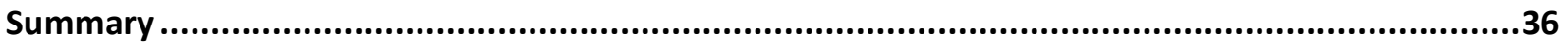

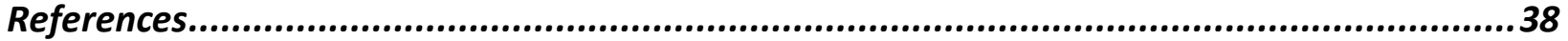

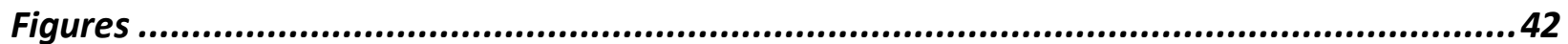

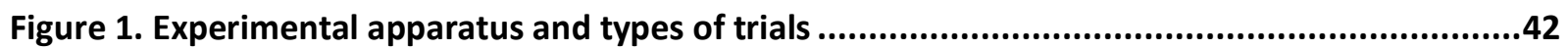

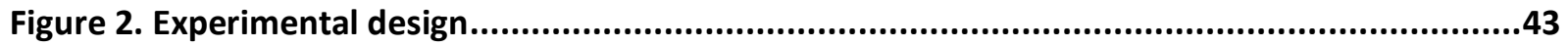

Figure 3. Sequence of events within each trial type..................................................................44

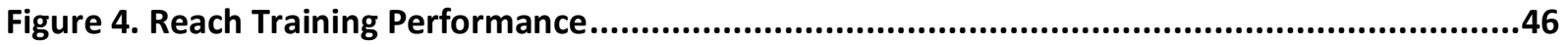

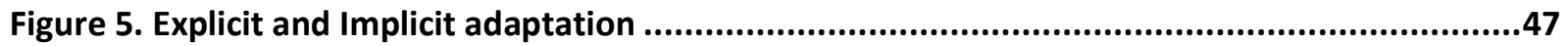

Figure 6. Adaptation indices as a percentage of reach adaptation .................................................48

Figure 7. Relationship between methods of assessment...........................................................49

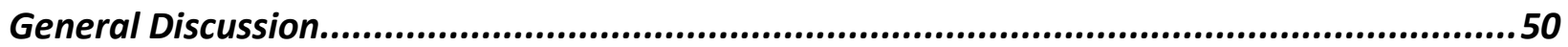

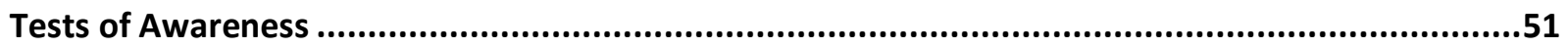

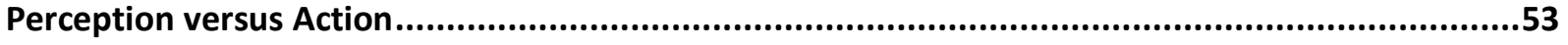

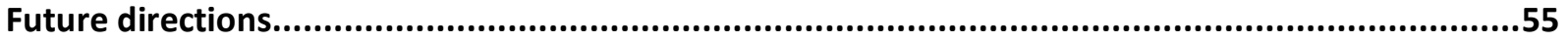

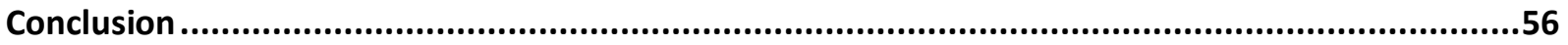

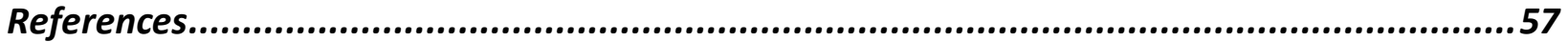

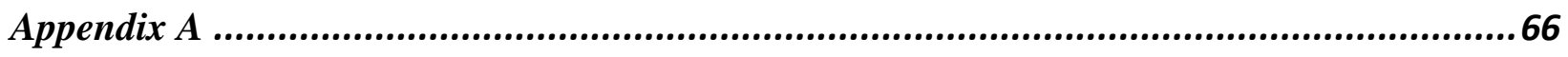

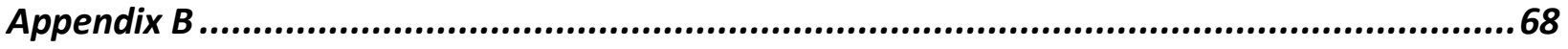

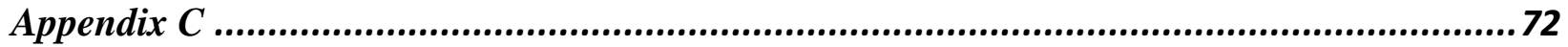

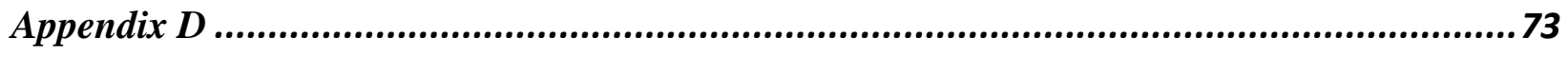

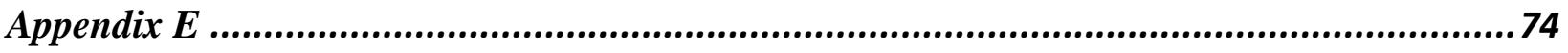

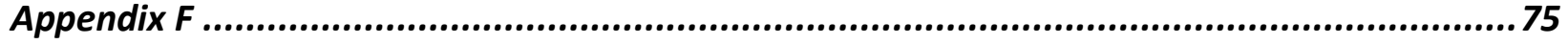

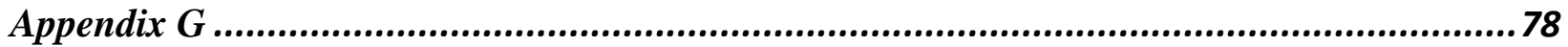


Running Head: EXPLICIT AND IMPLICIT VISUOMOTOR ADAPTATION

\section{List of Figures}

Figure 1. Experimental apparatus and types of trials

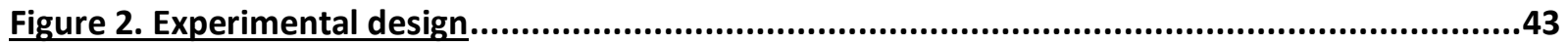

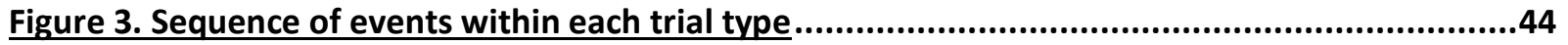

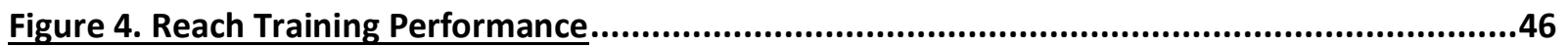

Figure 5. Explicit and Implicit adaptation ...............................................................................47

Figure 6. Adaptation indices as a percentage of reach adaptation ............................................48

Figure 7. Relationship between methods of assessment.............................................................49 
Running Head: EXPLICIT AND IMPLICIT VISUOMOTOR ADAPTATION

\section{Abstract}

The Process Dissociation Procedure (PDP) and Verbal Report Framework (VRF) have demonstrated that both explicit (Explicit Adaptation, EA) and implicit processes (Implicit Adaptation, IA) contribute to visuomotor adaptation. However, the definition of EA is inconsistent across the two paradigms, such that the PDP refers to EA as reflecting one's knowledge regarding how they have to reach in the novel visuomotor environment, while the VRF refers to EA as reflecting pre-planned aiming strategies. The objective of the current experiment was to compare EA as assessed via the PDP and VRF and hence provide insight into if they are assessing similar explicit processes. Sixty-one participants were evenly divided into three groups (PDP, VRF and VRF-No Cursor) and trained to reach in a virtual environment with an aligned cursor (1 block of 45 trials) and then a cursor rotated $40^{\circ}$ clockwise $(\mathrm{CW})$ relative to hand motion (3 blocks of 45 trials). EA and IA were assessed immediately following each block of rotated reach training trials, and again 5-minutes later. In the assessment trials, the PDP group reached while using any learned strategy (EA+IA), or while not engaging in a strategy (IA) and the VRF group reported their planned aiming direction by picking a number from an array of numbers surrounding the target (EA), before reaching to the target (EA+IA) with visual feedback. The VRF-No Cursor group completed the same assessment trials as the VRF group, but no visual feedback was presented during assessment of EA and IA. Following this, participants completed a post-experiment questionnaire and a drawing task to assess their awareness of the visuomotor rotation and changes in their reaches respectively. We found that all groups adapted their reaches to the $40^{\circ} \mathrm{CW}$ cursor rotation. As well, averaged across participants, the magnitude and retention of EA and IA were similar between the PDP and VRF groups. However, the magnitude of EA established via the VRF was not related to participants' post-experiment awareness of the visuomotor distortion and how they had changed their reaches, as observed in the PDP and VRF No-Cursor groups. Together, 


\section{Running Head: EXPLICIT AND IMPLICIT VISUOMOTOR ADAPTATION}

these results indicate that, while the PDP and VRF suggest similar contributions of EA and IA to visuomotor adaptation, the methods of assessment engage different explicit processes. EA assessed within the VRF does not reflect one's awareness of the visuomotor distortion at the end of the experiment or how they changed their reaches. 


\section{Chapter I: Literature Review}

\section{Goal Directed Reaching}

Simple reaching movements to a visual target (e.g., reaching for a coffee-mug or a pen), require complex sensorimotor transformations involving many levels of the central nervous system (CNS; Jeannerod, 1988). The CNS processes visual and proprioceptive information related to the limb's position and orientation, as well as visual information related to the target's position in order to plan and execute movements correctly. It has been hypothesized that the CNS creates an internal representation of the sensorimotor system, termed an internal model, in order to facilitate the transformation of incoming sensory information to appropriate motor commands (Francis \& Wonham, 1976; Wolpert \& Ghahramani, 2000; Wolpert, Ghahramani, \& Jordan, 1995). The internal model consists of two distinct parts: the inverse model and the forward model (Wolpert et al., 1995). The inverse model consists of the motor plan required to produce the desired action based on current visual and proprioceptive sensory information regarding target and limb positions (Desmurget \& Grafton, 2000). The forward model predicts the sensory consequences of the planned movement. Incoming sensory information is then compared to the predicted sensory consequences and, should sensory discrepancies arise, for example due to a change in visuomotor mapping, an error signal is generated. The motor plan (i.e., inverse model) and expected sensory consequences (i.e., forward model) are then updated accordingly in subsequent movements to minimize future errors (Wolpert et al., 1995).

\section{Visuomotor Adaptation}

Novel visuomotor mappings are commonly experienced. For example, when using your friend's computer, you may need to adapt your movements to a different mouse or trackpad setting. As well, movements will have to be adapted when putting on a pair of glasses with a new 
Running Head: EXPLICIT AND IMPLICIT VISUOMOTOR ADAPTATION

prescription and/or reaching for an object under water, as the visual consequences of the movement have changed relative to a typical environment. When reaching in a novel visuomotor environment, one initially experiences errors (e.g., reaches may not land on the target). However, the motor system adapts to the novel visuomotor environment, and errors are reduced to baseline levels (i.e., errors are similar to when reaching in a typical visual environment; Baraduc \& Wolpert, 2002; Simani et al., 2007; Cressman \& Heneiques, 2009). This visuomotor adaptation has been shown to arise quickly, within as little as 20 to 30 trials (Maksimovic \& Cressman, 2018; Krakauer et al., 2000).

Visuomotor adaptation has been defined as a type of motor learning, as improvements in performance are seen following practice in the altered environment (Krakauer, 2009; Bastian, 2008; Martin, Keating, Goodkin, Bastian, \& Thach, 1996). As well, continued improvements are observed across practice sessions, such that movements are adapted more quickly in subsequent practice sessions (Huang, Haith, Mazzoni, \& Krakauer, 2011; Krakauer, Ghez, \& Ghilardi, 2005; Morehead, Qasim, Crossley, \& Ivry, 2015; Zarahn, Weston, Liang, Mazzoni, \& Krakauer, 2008). Finally, there is evidence that these adapted movements are persistent, in that participants continue to reach with the adapted movement even when returning to reach in a normal, typical environment (Buch, Young \& Contreras-Vidal, 2003; Krakauer et al., 2000; Kitazawa, Kimura \& Uka, 1997). Specifically, participants show evidence of aftereffects, such that they continue to reach as they did in the altered visual environment after the visuomotor distortion is removed. This persistency in action has been taken as further evidence that visuomotor adaptation is a form of motor learning (Bastian, 2008).

The phenomenon of visuomotor adaptation has been examined in laboratory settings by modifying participants' visual environments through the use of laterally displacing prism goggles 
Running Head: EXPLICIT AND IMPLICIT VISUOMOTOR ADAPTATION

(Harris, 1963; Welch, Bridgeman, Anand, \& Browman, 1993; Redding \& Wallace, 1978, 1993, 1994, 1996, 2002, 2003, 2006) or having participants reach in a virtual reality environment while misrepresenting where their hand is in space via a cursor on the screen (Krakauer et al., 1999; Krakauer et al., 2000; Ghahramani, Wolpert, \& Jordan, 1996; Cressman \& Henriques, 2009). In general, participants complete three phases of testing: a pre-test or baseline phase, an adaptation or training phase, and a post-test. In the pre-test, participants reach in a typical environment (i.e., without the prism goggles, or when a cursor accurately represents where their hand is in space). During the adaptation phase, participants reach in the novel visuomotor environment, such that they put on the laterally displacing prism goggles or a cursor on the screen misrepresents where their hand is in space. In order for the visual representation of their hand to land on the target, participants must adapt their movements. For example, if the trajectory of the cursor on the screen is rotated $40^{\circ}$ clockwise $(\mathrm{CW})$ relative to hand motion, participants must aim $40^{\circ}$ to the left of the target. In the post-test, conditions are typically similar to that in the pre-test, such that participants reach in the absence of the prism goggles or when the cursor's position accurately represents where their hand is in space (Kitazawa et al., 1997; Buch et al., 2003; Wang \& Lei, 2015), or when no visual feedback of their hand position is provided (Cressman \& Henriques, 2009; Ghahramani, Wolpert \& Jordan, 1996; Werner et al., 2015; Poh, Caroll, \& Taylor, 2016).

Across these 3 phases of testing, large reaching errors are typically seen at the start of the adaptation phase, and again during the post-test. Specifically, early on during the adaptation phase, participants reach directly to where they see the target. This creates an error, as in the case of our virtual reality environment, the visual cursor misrepresents where the hand is in space and hence does not go to the target like the hand does (e.g., the cursor's trajectory is rotated $40^{\circ} \mathrm{CW}$ relative to the hand and hence target). Participants quickly adapt to these errors, such that they begin to 


\section{Running Head: EXPLICIT AND IMPLICIT VISUOMOTOR ADAPTATION}

aim left of where they see the target. In the post-test, participants continue to reach to the left of the target, like they did at the end of the adaptation phase. Thus, if the cursor is present it will be seen to fall left of the target, as its trajectory is aligned with the participant's actual reaching trajectory. If the cursor is not present, participants will continue to reach to the left of the target, not receiving visual feedback that their hand does not land on the target (Bond \& Taylor, 2015; Cressman \& Henriques, 2009; Ghahramani et al., 1996; Werner et al., 2015). The changes in reaches in the post-test compared to the pre-test are referred to as aftereffects and are taken as evidence that the internal model has been adapted.

\section{Processes underlying visuomotor adaptation}

Visuomotor adaptation has traditionally been modeled as a form of error-based learning (Martin et al., 1996). Specifically, participants experience a sensory prediction error, such that the visual representation of their hand does not land on the target as expected (Smith, Ghazizadeh \& Shadmehr, 2006; Taylor, Wojaczynski \& Ivry, 2011; Taylor et al., 2014; McDougle et al., 2015), and update their internal model accordingly. This error-based learning is proposed to arise implicitly (i.e., unconsciously), giving rise to the aftereffects observed in the post-test even when participants are instructed to aim directly to the target and/or the visuomotor distortion is turned off (Bond \& Taylor, 2015).

In addition to implicit processes, explicit processes have been suggested to underlie visuomotor adaptation. In fact, early work by Redding and Wallace (1978) proposed a role for explicit and implicit processes in visuomotor adaptation to laterally displacing prism goggles. Throughout a volume of work, they suggest that the changes in reaches observed initially, when the prisms are first put on, are driven by explicit processes, whereas implicit processes contribute

to visuomotor adaptation later in reach training (Redding \& Wallace, 1996, 2001, 2002, 2006). 


\section{Running Head: EXPLICIT AND IMPLICIT VISUOMOTOR ADAPTATION}

For example, movements are first altered explicitly during the adaptation phase while wearing laterally displacing prism goggles due to conscious strategic processes that coordinate perception and action (i.e., strategic perceptual-motor control), to reduce movement errors in order to reach the target accurately. After these initial, explicit corrections, participants continue to adapt their movements by engaging unconscious, implicit processes, which give rise to the realignment of visual and proprioceptive maps (i.e., adaptive spatial re-alignment).

Distortion of the visual environment through prism goggles differs from visuomotor distortions presented via a cursor on the screen in a virtual reality environment. In particular, prisms shift all visual input (e.g., hand and target locations), whereas only visual feedback of the hand position is shifted in virtual reality environments (Henriques \& Cressman, 2012). As well, participants are aware that they are putting on or taking off the prism goggles, creating awareness that a change in task is being introduced. Similar contextual cues, as provided in the prism adaptation studies, are not always present when reaching in a virtual reality environment. Nevertheless, findings suggest that reaches in a virtual reality environment are also adapted in response to a visuomotor distortion due to multiple learning processes, and that these processes are engaged in a similar timeframe as the strategic perceptual-motor control and adaptive spatial re-alignment processes outlined by Redding and Wallace (1996, 2001, 2002, 2006).

Smith and colleagues (2006) reported that fast and slow processes are responsible for adaptation of movement dynamics when reaching in a velocity-dependent force field. According to Smith and colleagues, the fast and slow processes operate in parallel, but with different timescales. The fast process is proposed to contribute to reach adaptation quickly by reducing initial direction errors. In contrast, the slow process allows participants to gradually reduce their movement errors over trials. Smith and colleagues' model has been shown to accurately predict 
Running Head: EXPLICIT AND IMPLICIT VISUOMOTOR ADAPTATION

reach adaptation patterns observed in visuomotor adaptation paradigms and the fast and slow processes have been suggested to map onto explicit and implicit processes, respectively (McDougle et al., 2015). This raises the question: "What explicit processes are engaged during visuomotor adaptation when visual feedback of the hand position is distorted within a virtual reality environment?"

\section{Explicit processes in visuomotor adaptation across methods of assessment}

Early attempts to examine explicit and implicit contributions to visuomotor adaptation used post-experiment questionnaires to divide participants into aware versus unaware groups (Benson, Anguera \& Seidler 2011; Hwang, Smith, \& Shadmehr, 2006; Heuer \& Hegele, 2008). Typically, after the completion of the experiment, participants were asked whether they noticed the task get harder or change at any point during the experiment. As well, participants were asked to describe the nature of the change and how they reached to counteract this change in order to complete the required task successfully. Thus, according to research involving post-experiment questionnaires, explicit processes encompass awareness of the visuomotor distortion and knowledge regarding how to reach in order to counteract the distortion that participants can verbally report.

Drawing tasks have also been used to assess the role of explicit processes in visuomotor adaptation and to probe one's awareness of a visuomotor distortion (Larssen, Ong, \& Hodges, 2012; Ong, Larssen, \& Hodges, 2012). Like the post-experiment questionnaires, drawing tasks are typically completed at the end of the experiment. Participants are removed from the original experimental room/setting and are allowed to see their limbs. Participants are then asked to use a pen (and perhaps a ruler) to draw the trajectory their hand took when they were training with the visuomotor distortion. Similar to the questionnaire data discussed above, the drawn hand trajectory 
Running Head: EXPLICIT AND IMPLICIT VISUOMOTOR ADAPTATION

error is interpreted as explicit adaptation, which reflects one's awareness of the visuomotor distortion and how one had to change their movements.

The definition of explicit processes is similar across these two post-experiment methods of assessment, even though one requires a verbal (perceptual) report, and the other a motor response. Moreover, the two tasks only assess explicit processes, not implicit. Based on post-experiment reports, participants are typically classified as aware (e.g., reported or demonstrated that they used a strategy to counteract the rotated visual feedback by reaching to the right of the target), or unaware (e.g., reported or demonstrated that they did not use a strategy to overcome the rotated visual feedback and reached to the target) of the visuomotor distortion. Performance is then compared between the aware and unaware groups of participants to establish the role of explicit and implicit processes in visuomotor adaptation, respectively. For example, using the postexperiment questionnaire, Benson et al. (2011) categorized participants into aware and unaware groups of participants. Results indicated that the aware group reached more accurately to the target at the beginning of the reach training trials with a visuomotor distortion, such that their errors were closer to baseline levels compared to the unaware group. The aware group also took longer to initiate their movements (i.e., had a longer reaction time).

While the post-experiment questionnaire and drawing task are able to assess participants' awareness of the visuomotor distortion and changes in their reaches at the end of the experiment, they are limited with respect to their ability to establish, and hence define, the role of explicit processes in visuomotor adaptation. Explicit and implicit processes have been shown to be independent, simultaneously contributing to visuomotor adaptation (Neville \& Cressman, 2018; Taylor \& Ivry, 2014; Werner et al., 2015). In addition, both explicit and implicit adaptation have been shown to be present in participants classified as aware or unaware of the visuomotor 
Running Head: EXPLICIT AND IMPLICIT VISUOMOTOR ADAPTATION

distortion at the end of the experiment (Neville \& Cressman, 2018; Taylor et al., 2014; Werner et al., 2015). Therefore, a binary task does not capture the contribution of explicit and implicit processes to visuomotor adaptation, nor does awareness of the visuomotor distortion at the end of the experiment necessarily encompass all explicit processes engaged during visuomotor adaptation.

Two methods have recently been put forward to establish the role of explicit and implicit processes to visuomotor adaptation; (1) the process dissociation procedure (PDP) and (2) the verbal report framework (VRF). As outlined below, the methods have subtle differences with respect to their definition of explicit processes and manner of assessment. The PDP was first introduced to the field of cognitive psychology by Jacoby (1991) as a methodological tool to measure the contributions of automatic and intentional memory to recognition. Since its introduction, the PDP method has become an accepted method in the cognitive domain for its ability to assess explicit and implicit processes (Destrebecqz \& Cleeremans, 2001; Bergerbest \& Goshen-Gottstein, 2001; Destrebecqz et al., 2003; Norman, Price \& Duff, 2006). This method was recently modified by Werner and colleagues (2015) to examine the contributions of explicit and implicit processes to visuomotor adaptation, where explicit processes are defined as knowledge (i.e., awareness) of how to reach in the novel visuomotor environment, as assessed within a motor task. Using the PDP, Werner and colleagues instructed participants to reach under exclusion (1) reach and "do not use any strategy you learned throughout training" (implicit adaptation) and inclusion (2) reach and "use the strategies you have learned throughout training" (explicit + implicit adaptation) instructions. Explicit adaptation is defined as the difference in reaching errors between inclusion and exclusion trials, whereas implicit adaptation reflects reaching errors on exclusion trials relative to the target (Modchalingam, Vachon, Hart \& Henriques, 2019; Neville \& 
Running Head: EXPLICIT AND IMPLICIT VISUOMOTOR ADAPTATION

Cressman, 2018; Werner, Strüder \& Donchin, 2019; Werner et al., 2015). Neville and Cressman have also used the PDP to examine the contributions of explicit and implicit processes to visuomotor adaptation over time. Together, Werner et al. (2015) and Neville and Cressman (2018) have shown that, in general, explicit adaptation is greater in participants that are given a strategy on how to counteract the visuomotor rotation, while implicit adaptation is greater in participants who are not given a strategy on how to counteract the visuomotor distortion. As well, implicit adaptation takes time to develop and decays following a 5-minute break. Finally, the sum of explicit and implicit adaptation has been shown to account for a varying percentage of reach adaptation observed during the reach training trials across experiments (e.g., 55\% of visuomotor adaptation in Werner et al. (2015) versus 97\% of visuomotor adaptation in Neville and Cressman (2018)).

The verbal reporting method was introduced by Taylor and colleagues in 2014 to assess explicit and implicit contributions to visuomotor adaptation over time, where explicit adaptation is defined as pre-planned aiming strategies that participants are able to consciously report (i.e., verbalize). In this method, participants are required to verbally report where they plan to aim before they reach to a target. Specifically, during reach training trials with the cursor, participants are shown a visual display in which the target is surrounded by a number array (i.e., total of 63 numbers separated by $5.625^{\circ}$; the target is set at 0 and numbers span from 1 to 31 in the clockwise direction from the target and from -1 to -31 in the counter-clockwise direction from the target). Before each reach, participants report the number that they plan to reach to in order to get the cursor to the target. Explicit adaptation is the difference between target location and the reported number location, while implicit adaptation is the difference between the reported number location and actual location the hand reached. In accordance with results using the PDP, Taylor and 
Running Head: EXPLICIT AND IMPLICIT VISUOMOTOR ADAPTATION

colleagues have shown that explicit processes initially contribute to visuomotor adaptation, with minimal contribution from implicit processes (Taylor et al., 2014; McDougle, Bond, Taylor, 2015). However, over reach training trials, implicit adaptation increases, until reaching a maximum value of approximately $11^{\circ}$, regardless of the size of the visuomotor distortion (Bond \& Taylor, 2015). The magnitude of explicit adaptation has been shown to be flexible, increasing with greater visuomotor rotations (Bond \& Taylor, 2015), and transferring between limbs (Poh et al., 2016). By design, explicit and implicit contributions sum to the extent of reach adaptation observed when reaching with the cursor.

\section{Rationale and Current Research Objectives}

The definition of explicit adaptation (EA) varies between the PDP and VRF methods of assessment, while the definition of implicit adaptation (IA) is consistent across paradigms (i.e., changes in reaches that arise due to unconscious and non-intentional changes to the internal model as a result of experiencing a sensory prediction error; Wolpert \& Kawato, 1998). The PDP method refers to EA as reflecting knowledge of how to reach in the novel visuomotor environment, as established via a motor task, while the VRF method refers to EA as reflecting consciously accessible, pre-planned aiming strategies. In the current project we looked to compare EA (and IA) as established via the PDP and VRF methods of assessment to provide insight into if they are assessing similar explicit processes.

Previous research using the PDP (e.g., Werner et al., 2015; 2019; Neville \& Cressman, 2018; Modchalingam et al., 2019) and VRF (e.g., Taylor, Ivry \& Krakauer, 2014; McDougle, Bond, \& Taylor, 2015; Poh, Carroll, \& Taylor, 2016; Taylor \& Ivry, 2011) have found similar trends with respect to the magnitude and time at which explicit and implicit processes contribute to visuomotor adaptation. For example, within both paradigms, EA has been shown to be 
Running Head: EXPLICIT AND IMPLICIT VISUOMOTOR ADAPTATION

responsible for reducing errors at the beginning of reach adaptation, while IA contributions increase over time (Taylor, Krakauer, \& Ivry, 2014; Werner et al., 2015). As well, explicit contributions increase with larger visuomotor distortions, whereas implicit contributions are consistent in magnitude regardless of rotation size across both methods of assessment (Bond \& Taylor, 2015; Neville \& Cressman, 2018; Werner et al., 2015). That said, there are also marked differences in results when comparing findings from the two paradigms. Specifically, the overall extent to which EA and IA contribute to visuomotor adaptation appears to differ between paradigms (ranging from 55\% - 97\% of reach adaptation observed using the PDP to $100 \%$ using the VRF). As well, explicit adaptation has been shown to play a greater role in intermanual transfer when assessed via the PDP compared to the VRF (Bouchard \& Cressman, 2017; Poh et al., 2016). Finally, retention of EA and IA have not been established within the VRF.

To date, explicit and implicit processes as established via the PDP and VRF can only be compared across experiments that differ with respect to visuomotor distortion size, number of reach training trials, time of assessment, etc. Thus, it is difficult to make conclusions on whether similar trends in EA and IA exist between paradigms, and hence similar processes are being examined. In order to determine if similar explicit processes are assessed within the PDP and VRF, we looked to compare EA (and IA) as established via the PDP and VRF methods of assessment within the same experiment.

Within the current experiment, EA and IA were assessed in 3 groups. For all groups, EA and IA were assessed immediately after completing a block of 45 rotated reach training trials, and again after a 5-minute break. Participants completed a total of 3 bocks of 45 rotated reach training trials, and hence EA and IA were assessed 6 times. In the first group, EA and IA were assessed using the PDP of Werner et al. (2015), such that participants reached in the absence of visual 
Running Head: EXPLICIT AND IMPLICIT VISUOMOTOR ADAPTATION

feedback when instructed to use any strategy they had learned (inclusion trials) and not use any strategy (exclusion trials). The second group completed trials in accordance with the established VRF method of assessment of Taylor and colleagues (2014), such that they first verbally reported where they planned to reach and then reached with the cursor present. We also included a third group of participants, the VRF No-Cursor group, who completed the VRF assessment trials while the cursor was not available. The inclusion of the third group allowed us to determine the role of visual feedback in the VRF paradigm, and if it may drive differences between EA and IA as established using the PDP versus VRF. At the end of the experiment, all participants completed the post-experiment questionnaire (PEQ) of Benson et al. (2011) and a drawing task (DT; adapted from Larssen et al., 2012), to assess their awareness of the visuomotor distortion and how they had to reach to counteract the visuomotor distortion at the end of the experiment.

We hypothesized that all participants would adapt their movements to the $40^{\circ} \mathrm{CW}$ visuomotor rotation, such that reaching errors at peak velocity would shift in order to counter the rotation so that the hand landed on the target (Ghahramani et al., 1996; Kagerer et al., 1997; Krakauer et al., 2000; 1999). We further expected that similar trends in EA and IA with respect to magnitude and retention would be observed between the PDP and VRF groups, indicating that the two methods assess similar explicit and implicit contributions, and hence, potentially similar explicit processes. Alternatively, if EA and/or IA differ based on manner of assessment, it would suggest that the two methods differ in the processes they are assessing. Finally, by examining EA in light of post-experiment responses, we can determine if EA as established via the PDP or VRF is available for conscious recall at the end of the experiment. In other words, does knowledge regarding how one has to reach as assessed by a motor task and one's consciously accessible pre- 
Running Head: EXPLICIT AND IMPLICIT VISUOMOTOR ADAPTATION

planned aiming strategy, reflect one's awareness of the visuomotor distortion and changes in their reaches at the end of the experiment?

\section{Chapter II: Research Article}

Assessing and defining explicit processes in visuomotor adaptation

Heirani Moghaddam ${ }^{1}, \mathrm{~S}, \mathrm{Chua}^{2}, \mathrm{R}, \mathrm{Cressman}^{1}$, EK

${ }^{1}$ Sensorimotor Control Laboratory, School of Human Kinetics, Faculty of Health Sciences, University of Ottawa, Ottawa, ON, K1N 6N5, Canada.

${ }^{2}$ Perceptual-Motor Dynamics Laboratory, School of Kinesiology, Faculty of Education, University of British Columbia, Vancouver, BC, V6T 1Z1, Canada.

Keywords:

Visuomotor Adaptation, Process Dissociation Procedure, Verbal Report Framework, Explicit, Implicit 
Running Head: EXPLICIT AND IMPLICIT VISUOMOTOR ADAPTATION

\section{Introduction}

When reaching in a novel visuomotor environment, one initially experiences reaching errors, such that the cursor does not go directly to the target (e.g., it lands to the right of the target). However, within a few trials, the motor system adapts to the visuomotor distortion, such that participants begin to aim to the left of the target, and the cursor once again lands on the target (i.e., errors are similar to those observed when reaching in a typical visual environment, in which the cursor is aligned with the hand; Cressman \& Henriques, 2009; Huang, Haith, Mazzoni, \& Krakauer, 2011; Krakauer et al., 2004; Krakauer, Ghilardi, \& Ghez, 1999; Neville \& Cressman, 2018; Krakauer, Pine, Ghilardi, \& Ghez, 2000; Mazzoni \& Krakauer, 2006; Zarahan, Weston, Liang, Mazzoni, Krakauer, 2008). Following these reach training trials, if the cursor is now removed and participants are instructed to aim to the target, they continue to reach to the left of the target. These aftereffects are proposed to arise implicitly and reflect changes to the internal model (Baraduc \& Wolpert, 2002; Buch, Young, \& Contreras-Vidal, 2003; Ghahramani et al., 1996; Wolpert \& Kawato, 1998).

Recently, it has been shown that explicit processes can also contribute to visuomotor adaptation (Benson, Anguera, \& Seidler, 2011; Bond \& Taylor, 2015; McDougle, Bond, \& Taylor, 2015; Neville \& Cressman, 2018; Taylor, Krakauer, \& Ivry, 2014; Werner, Strüder, \& Donchin, 2019; Werner et al., 2015). Specifically, two methods have been used to demonstrate that visuomotor adaptation can be driven by a combination of explicit and implicit processes at the same time: the Process Dissociation Procedure (PDP; Neville \& Cressman, 2018; Werner et al., 2015) and the Verbal Report Framework (VRF; Taylor et al., 2014). Within the PDP, participants complete a series of reach training trials with the visuomotor distortion. Following these reach training trials, they are then asked to reach when they (1) "do not use any strategy you learned" 
Running Head: EXPLICIT AND IMPLICIT VISUOMOTOR ADAPTATION

(i.e., exclusion trials) or (2) "use the strategies you have learned" (i.e., inclusion trials). Explicit adaptation is determined as the difference in reaching errors between exclusion and inclusion trials, while implicit adaptation is evaluated based on performance in the exclusion trials (Werner et al., 2015). In the VRF method, explicit and implicit adaptation are typically assessed during the reach training trials when a target is presented, with an array of numbers surrounding it. Participants first verbally report the number (i.e., location) they intend to aim to in order to get the cursor to the target and then reach with the goal of getting the cursor to the target (Taylor et al., 2014). Explicit adaptation is determined by reported aiming direction, whereas implicit adaption is taken as the difference between reaching errors and reported aiming direction.

At present, it is unclear if these two methods are measuring the same processes, specifically with respect to explicit contributions to visuomotor adaptation. While the definition of implicit processes is fairly consistent across the literature, regardless of method of assessment (i.e., unconscious changes in one's movements that arise in the absence of awareness or intention, that result in updating of an internal model), the definition of explicit processes changes based on method of assessment. The PDP refers to explicit processes as reflecting knowledge (i.e., awareness) of how one has to move in order to counteract the visuomotor distortion, where this knowledge can be turned on or off (Werner et al., 2019, 2015). In contrast, the VRF method refers to explicit processes as reflecting cognitive aiming strategies that are engaged in order to get the cursor to the target, without referring to awareness of the visuomotor distortion (Bond \& Taylor, 2015; McDougle et al., 2015; Taylor et al., 2014). In addition to differences in the definition of explicit processes being assessed, the two methods utilize different tasks to establish explicit and implicit contributions to visuomotor adaptation. The PDP method indirectly assesses explicit processes, as explicit adaptation is inferred based on subtracting reaching errors. In contrast, 
Running Head: EXPLICIT AND IMPLICIT VISUOMOTOR ADAPTATION

explicit processes are more directly assessed in the VRF, as participants verbally indicate their aiming strategy.

While patterns with respect to the magnitude and timing of explicit contributions to visuomotor adaptation are similar when comparing results achieved across research employing the PDP and VRF (e.g., see McDougle et al., 2015; Poh, Carroll, \& Taylor, 2016; Taylor et al., 2014; Werner et al., 2015), inconsistencies can also be found. Specifically, the overall extent to which explicit and implicit processes contribute to visuomotor adaptation differs between paradigms (e.g., ranging from 55 to $97 \%$ of visuomotor adaptation in the PDP (Werner et al., 2015; Bouchard \& Cressman, 2017; Neville \& Cressman, 2018; Modchalingam et al., 2019) to 100\% in the VRF). As well, explicit adaptation has been shown to play a greater role in intermanual transfer when assessed via the PDP compared to the VRF (Bouchard \& Cressman, 2017; Poh et al., 2016). Finally, the retention of explicit and implicit processes has not been established within the VRF. Thus, it is unclear if the PDP and VRF are assessing the same (explicit) processes underlying visuomotor adaptation.

The goal of this study was to determine the relationship between explicit processes as assessed via the PDP and VRF. To do this, we compared the extent of explicit and implicit adaptation over time when assessed via the PDP and VRF. All participants adapted to a visuomotor distortion during 3 consecutive blocks of 45 reach training trials, in which the cursor was rotated $40^{\circ}$ clockwise relative to hand motion. Explicit and implicit processes were immediately assessed after each block of reach training trials, and again after a 5-minute delay. A third group of participants (VRF No-Cursor group), was also included in this experiment. The VRF No-Cursor group received similar instructions as the VRF group but did not receive visual feedback regarding their reaching movements during the assessment trials, enabling us to examine the role of visual 
Running Head: EXPLICIT AND IMPLICIT VISUOMOTOR ADAPTATION

feedback during VRF assessment. Finally, we used Benson and colleagues' (2011) postexperiment questionnaire and a drawing task (see Larssen, Ong, \& Hodges, 2012; Ong \& Hodges, 2010) in order to probe participants' awareness of changes in their reaches at the end of the experiment. We hypothesized that all participants would adapt their reaches to the $40^{\circ} \mathrm{CW}$ visuomotor rotation during reach training trials, such that the cursor would land on the target. Similar extents of explicit and implicit adaptation over time would indicate that the PDP and VRF assess similar processes underlying visuomotor adaptation. Alternatively, if explicit and/or implicit adaptation differ based on manner of assessment, it would suggest that the two methods differ in the processes they are assessing (e.g., awareness regarding how one has to reach as assessed by a motor task does not correspond to one's preplanned consciously accessible aiming strategy).

\section{Methods}

\section{Participants}

61 naïve participants, aged $19-40(\mathrm{M}=24$ years, $\mathrm{SD}=0.03)$ were recruited from the University of Ottawa community and randomly divided into 3 groups; (1) PDP (N = 21), (2) VRF $(\mathrm{N}=20)$, (3) VRF No-Cursor $(\mathrm{N}=20)$ groups. All participants had normal or corrected to normal vision, were right handed according to the Edinburgh handedness questionnaire $(\mathrm{M}=81, \mathrm{SD}=$ 3.2; Oldfield, 1971) and self-reported no history of motor, sensory or cognitive impairment. This project was approved by the University of Ottawa Research Ethics Board, and participants provided informed consent prior to testing.

\section{Apparatus}

Participants reached to visual targets using the BKIN KINARM End-Point Lab (BKIN Technologies, Kingston, ON). As shown in Figure 1, visual targets were projected from a 
Running Head: EXPLICIT AND IMPLICIT VISUOMOTOR ADAPTATION

downward facing monitor, located $20.5 \mathrm{~cm}$ above a reflective surface that was located $20.5 \mathrm{~cm}$ above the robot handle. Thus, visual stimuli appeared to lie in the same horizontal plane as the right reaching hand holding on to the robot handle. Participants' view of their limbs was occluded by the reflective surface and a black cloth that was attached to the experimental apparatus and secured around participants' necks. Once participants were comfortably seated, the lights in the room were turned off, and testing began.

\section{Experiment overview}

In general, participants reached from a home location positioned $20 \mathrm{~cm}$ in front of their midline (white circle, $2 \mathrm{~cm}$ in diameter; Figure 1) to one of three visual targets located $15 \mathrm{~cm}$ from the home position, at positions straight ahead $\left(0^{\circ}\right.$ target $)$ and $45^{\circ}$ to the left and right of center (yellow circles, $1 \mathrm{~cm}$ in diameter; Figure 1B). Visual feedback regarding participants' hand location was presented on some trials in the form of an on-screen cursor (magenta circle; $1 \mathrm{~cm}$ in diameter). Participants began by completing 9 practice trials to become comfortable with the testing apparatus. They then completed 4 testing blocks within a single testing session (Figure 2). Within each testing block, there was 1 set of 45 reach training trials, followed by 2 sets of 12 trials to assess explicit and implicit visuomotor adaptation. The 2 sets of 12 trials were separated by 5 minutes, to establish the persistence of explicit and implicit adaptation.

Once all reaching trials were completed, participants exited the testing room. They then completed a questionnaire designed to assess their ability to verbally report their awareness of the visuomotor distortion (questionnaire from Benson et al., 2011). Finally, participants completed a drawing task that was similar in nature to the one used by Larssen and colleagues (2012). In the drawing task, the home position and three targets were drawn to scale on a sheet of paper. Participants were asked to draw the trajectory their hand took to get the cursor to the target during 
Running Head: EXPLICIT AND IMPLICIT VISUOMOTOR ADAPTATION

the Rotated blocks described below. Their hand was visible during these trials. The entire testing session lasted approximately one hour and thirty minutes.

Trial types

Reach Training Trials

Similar instructions were provided to all participants during the reach training trials, regardless of group (Figure 2).

\section{Aligned Cursor Reach Training Trials}

The Aligned cursor Reach Training trials began with the robot moving a participant's hand into the home circle (Figure 1C). Following $500 \mathrm{~ms}$, one of the three yellow targets appeared. Participants were instructed to wait until the yellow target turned blue (i.e., variable delay of 500$1200 \mathrm{~ms}$ ) and then reach to the target as quickly as possible, with the goal of landing the cursor on the target. Visual feedback regarding hand position was displayed in the form of a cursor on the screen and appeared once the hand moved out from the home position. A movement was defined as complete once velocity dropped below $0.01 \mathrm{~m} / \mathrm{s}$ and the hand was held at its final position for $500 \mathrm{~ms}$. At this time, the cursor and the target disappeared. Following another pause of $500 \mathrm{~ms}$, the robot passively moved the hand back to the home position in a movement time (MT) of 1000 ms. The robot handle followed a linear grooved path directly back to the home position and no visual feedback was provided. If participants attempted to move outside of the linear path, a resistance force (proportional to the depth of penetration with a stiffness of $2 \mathrm{~N} / \mathrm{mm}$ and a viscous dampening of $5 \mathrm{~N} / \mathrm{mm}$ ) perpendicular to the grooved path was produced. The position of the KINARM robot was recorded at a sampling rate of $1000 \mathrm{~Hz}$, with a spatial accuracy of $0.1 \mathrm{~mm}$.

\section{Rotated Cursor Reach Training Trials}

The timing of events during the Rotated cursor Reach Training trials was the same as the Aligned cursor Reach Training trials, in that participants were instructed to reach to the target as 
Running Head: EXPLICIT AND IMPLICIT VISUOMOTOR ADAPTATION

quickly as possible once it turned from yellow to blue. On these trials, however, the position of the cursor was misaligned from a participant's actual hand location, such that its motion was rotated $40^{\circ}$ clockwise $(\mathrm{CW})$ relative to a participant's hand trajectory (Figure 1D). Note that participants were not made aware of the rotation, in that they were not told of the visuomotor distortion, nor instructed on how to counteract it.

\section{Trials to assess Explicit and Implicit contributions to Visuomotor Adaptation} PDP Group: No Cursor Reach Trials

In the PDP no-cursor reach trials, participants reached to the target in the absence of visual feedback (i.e., no cursor was displayed). Similar to the reaches described above, participants waited until the target changed from yellow to blue and then reached to the target as quickly and accurately as possible. During the no-cursor reaches, participants reached under either exclusion or inclusion instructions. Specifically, each set of 12 PDP trials was split into 6 exclusion and 6 inclusion trials. The order in which exclusion and inclusion trials was completed was counterbalanced across participants.

In exclusion trials, participants were instructed as followed: "You are now going to reach when you cannot see your hand, as there will be no cursor on the screen. For these trials, do not use anything you may have learned to get the cursor to the target. Instead, aim so that your hand goes straight to the target as you did during baseline reaches". In inclusion trials, participants were instructed as follows: "You are now going to reach when you cannot see your hand, as there will be no cursor on the screen. For these trials, use anything you have learned or any strategies that you have developed during training in order to get the cursor to the target. In other words, aim so that the cursor would have gone straight to the target, as in the training trials you just completed" (Figure 1E). Note that following the aligned cursor reach training trials, participants completed all PDP trials with the exclusion trial instructions, such that they were to reach to the target. 
Running Head: EXPLICIT AND IMPLICIT VISUOMOTOR ADAPTATION

\section{VRF No-Cursor and VRF groups: VRF Trials}

In the VRF trials, the visual display changed (Figure 1F). A trial started with the hand positioned in the home position. One of the three yellow targets was then displayed, and a number array was also presented surrounding the target. Specifically, 46 numbers were presented on a semicircle at the same distance as the target from the home position. The numbers ranged from 23 to +23 (the target being at 0 ), and spanned $138^{\circ}$ in total, such that the numbers were separated by $3^{\circ}$. Participants were told that "The visual display will now change. You may have noticed that there are little numbers flanking the target. I would like you to tell me, before reaching, the number that you think you should aim to in order to get your cursor on the target. So, if you think that you should aim directly at the target, then please say "0". But if you think that you should aim somewhere else, please tell me what that number is." The experimenter recorded the participant's entry. After the verbal response was entered, the target changed from yellow to blue immediately and participants reached as in the reach training trials. The numbered array stayed on for the duration of the trial. In the VRF group, cursor feedback was presented, such that the cursor was either aligned or rotated relative to the hand motion, depending on the reach training block just completed. In the VRF No-Cursor group, the cursor was not presented during these trials.

\section{Data Analysis}

Results from the post-experiment questionnaire (PEQ), the drawing task (DT) and the assessment trials revealed that the majority of participants (45 out of 61 ) were aware of the visuomotor distortion and demonstrated explicit adaptation. The remaining 16 participants (PDP: 6 participants; VRF: 4 participants; VRF No-Cursor: 7 participants) were classified as unaware of the visuomotor distortion based on the PEQ and/or they failed to show explicit reach adaptation in the assessment trials. Given our question of interest, these participants were excluded from the 
Running Head: EXPLICIT AND IMPLICIT VISUOMOTOR ADAPTATION

analyses below. Secondary analyses including all 61 participants revealed similar findings to the results reported.

Reaching performance on all trials were analyzed using custom written programs in MATLAB. The start and endpoint of each reaching movement were defined using a velocity criterion. The point in time at which velocity first increased above or decreased below $0.01 \mathrm{~m} / \mathrm{s}$ for $50 \mathrm{~ms}$ was defined as movement onset and movement termination, respectively. For each of the reaching profiles, we determined reaction time (i.e., RT: from the time the target changed to blue to movement onset), movement time (i.e., MT: interval of time between movement onset and movement termination), and the angular error at peak velocity (i.e., PVAE: the angular difference between a movement vector from the home position to peak velocity and a reference vector joining the home position to the target).

We first looked to establish if participants adapted to the $40^{\circ} \mathrm{CW}$ cursor rotation during the reach training trials. PVAE were averaged over the first (time 1) and last 6 (time 2) reach training trials within each of the 4 reach training blocks (i.e., 1 block with an aligned cursor and 3 blocks with a rotated cursor). From these average errors, initial and later performance errors when reaching with an aligned cursor (block 1) and rotated cursor (blocks 2, 3 and 4) were compared using a 3 group (PDP, VRF, VRF No-Cursor) x 4 block (block 1 aligned, block 2 rotated, block 3 rotated, block 4 rotated) $\times 2$ time (mean of $1^{\text {st }}$ bin of 6 trials vs. last bin of 6 trials) mixed analysis of variance (ANOVA), with repeated measures (RM) on the last two factors. RT and MT were analyzed in a similar manner.

Following this, explicit and implicit contributions to visuomotor adaptation were determined from the PDP and VRF assessment trials. For the PDP trials, mean PVAE for each set of 6 exclusion or inclusion trials were determined. Explicit and Implicit Indexes were then 
Running Head: EXPLICIT AND IMPLICIT VISUOMOTOR ADAPTATION

determined according to the following formulas for each of the 8 times that PDP trials were performed (i.e., immediately and 5 minutes after each block of reach training trials):

PDP trials:

Explicit Index $=M_{\mathrm{PVAE} \text { on Inclusion trials }}-M_{\mathrm{PVAE}}$ on Exclusion trials

Implicit Index $=M_{\mathrm{PVAE}}$ on Exclusion trials

For the VRF trials, an explicit and an implicit index were calculated for each trial according to the following formulas:

VRF trials:

Explicit Index $=$ Verbal Report $\times 3^{\circ}$

Implicit Index $=P V A E-$ Explicit index

Explicit and Implicit indexes were then averaged across the 12 trials within each block of VRF assessment trials, resulting in 8 Explicit and Implicit indexes.

Initial analysis established that Explicit and Implicit Indexes for all groups differed following training with the rotated cursor compared to the aligned cursor. Thus, explicit adaptation (EA) and implicit adaptation (IA) following the rotated training blocks were calculated by subtracting a participant's Explicit index or Implicit index from the corresponding aligned block. To determine if the EA or IA differed across methods of assessment, we performed separate 3 group (PDP, VRF, VRF No-Cursor) x 3 block (block 2 rotated, block 3 rotated, block 4 rotated) x 2 time (immediately following reach training trials vs. following a 5 minute delay) mixed ANOVA with RM on the last two factors. RT and MT were compared across the VRF and VRF No-Cursor groups using a 2 group (VRF No-Cursor, VRF) x 4 block (block 1 aligned, block 2 rotated, block 3 rotated, block 4 rotated) $\times 2$ time (immediately following reach training trials vs. following a 5minute delay) mixed ANOVA with RM on the last two factors. The PDP group was not included 
Running Head: EXPLICIT AND IMPLICIT VISUOMOTOR ADAPTATION

in these analyses given that they were not required to report a number prior to reaching. Instead, to determine if there were differences in RT and MT between exclusion and inclusion trials, a 2 trial type (exclusion vs. inclusion) x 4 block (block 1 aligned, block 2 rotated, block 3 rotated, block 4 rotated) x 2 time (immediately following reach training trials vs. following a 5-minute delay) ANOVA with repeated measures on all 3 factors was performed.

After analyzing EA and IA, the percentage of reach adaptation that could be attributed to EA and IA was calculated for each group. First, the sum of EA and IA for each participant, in each of the blocks at time 1 (immediately after rotated reach training) and time 2 (after the 5-minute delay) were determined. These values were then divided by the extent of reach adaptation observed in the reach training trials (i.e., PVAE of the last 6 reach training trials within a given rotated block - PVAE of the last 6 reach training trials within the aligned block), such that the extent of reach adaptation accounted for by EA and IA was defined as:

$\%=\frac{(E A+I A)}{\text { Rotated training block }(\text { Bin } 2)-\text { Aligned training block }(\text { Bin } 2)} \times 100$

To determine if the percentage of reach adaptation attributed to EA and IA differed across groups, we performed a 3 group (PDP, VRF, VRF No-Cursor) x 3 block (block 2 rotated, block 3 rotated, block 4 rotated) $\times 2$ time (immediately following reach training trials vs. a 5-minute delay) mixed ANOVA with RM on the last two factors.

As well, for each group, we completed Pearson's correlation tests to determine the strength and direction of the relationship between our assessments of awareness (PEQ or DT) at the end of the experiment and EA in block 4 rotated, time 1.

The significance value for all statistical tests was set at $\mathrm{p}<.05$, and Bonferonni post-hoc tests corrected for multiple comparisons were used to find the locus of significant effects for all pre-planned comparisons. In addition, coefficients of determination $\left(\mathrm{R}^{2}\right)$, and slopes for linear 
Running Head: EXPLICIT AND IMPLICIT VISUOMOTOR ADAPTATION

regressions are reported to describe the relationship between the variables of interest where applicable. When appropriate, Greenhouse-Geisser correction was applied and reported.

\section{Results}

Awareness as assessed via the Post-Experiment Questionnaire (PEQ) and Drawing Task (DT)

Overall, PEQ responses $\left(\mathrm{M}=30.2^{\circ}, \mathrm{SD}=10.9^{\circ}\right)$ and trajectory deviations in the $\mathrm{DT}(\mathrm{M}=$ $22.8^{\circ} ; \mathrm{SD}=10.9^{\circ}$ ) revealed that participants who were included in the analyses were aware of the visuomotor distortion and changes in how they had to reach by the end of the testing session. Oneway ANOVA revealed that responses on the PEQ and DT did not differ between groups (PEQ: $F(2,42)=.275, p=0.761 ; \mathrm{PDP}: \mathrm{M}=31.5^{\circ}, \mathrm{SD}=10.9^{\circ} ; \mathrm{VRF}: \mathrm{M}=30.4^{\circ}, \mathrm{SD}=11.0^{\circ} ; \mathrm{VRF}$ NoCursor: $\mathrm{M}=28.3^{\circ}, \mathrm{SD}=11.5^{\circ} ; \mathrm{DT}: F(2,42)=.486, p=0.619 ; \mathrm{PDP}: \mathrm{M}=20.9^{\circ}, \mathrm{SD}=16.7^{\circ}$; VRF: $\mathrm{M}=24.8^{\circ}, \mathrm{SD}=4.97^{\circ}$; VRF No-Cursor: $\mathrm{M}=22.5^{\circ}, \mathrm{SD}=7.16^{\circ}$ ). Paired samples t-test between the PEQ and DT for each group revealed a significant difference between the PEQ and DT only in the PDP group $(p<0.001)$ but not in the VRF $(p=0.060)$ or the VRF No-Cursor group $(p=0.171)$

\section{Reach Training Trials}

Peak Velocity Angular Error (PVAE)

PVAE across the 4 blocks of reach training trials for the PDP, VRF and VRF No-Cursor groups are shown in Figure 4. From this figure, we see that participants' reaches were fairly accurate in block 1 aligned (M PVAE $=1.8^{\circ}, \mathrm{SD}=0.36^{\circ}$ to the left of the target), regardless of group. In block 2 rotated, when the $40^{\circ} \mathrm{CW}$ cursor rotation was first introduced, we see that participants' reaches began to deviate to counteract the rotation. ANOVA revealed a significant main effect of block $(F(3,40)=1134.6, p<0.001)$, and time $(F(1,40)=158.476, p<0.001)$, and significant interactions between block x time $(F(3,40)=722.508, p<0.001)$ and group $\mathrm{x}$ time 
Running Head: EXPLICIT AND IMPLICIT VISUOMOTOR ADAPTATION

$(F(2,40)=51.502, p=0.040)$. Post hoc analyses revealed that PVAE during all rotated blocks were greater than those in block 1 aligned. As well, the extent of adaptation increased over the rotated blocks from $25.25^{\circ}$ in block $2\left(\mathrm{SD}=0.76^{\circ}\right)$, to $32.53^{\circ}$ in block $3\left(\mathrm{SD}=0.64^{\circ}\right)$, to $34.79^{\circ}$ in block $4\left(\mathrm{SD}=1.10^{\circ}\right)$. Also, PVAE was greater at the end of each rotated block (i.e., time 2, last 6 trials) compared to the beginning of the same block (i.e., time 1, first 6 trials) in all rotated blocks, regardless of group. In accordance with the significant block $\mathrm{x}$ time interaction, post hoc analysis revealed that at time 1, participants' increased across blocks (block 1 aligned: $\mathrm{M}=1.71^{\circ}, \mathrm{SD}=$ $1.28^{\circ}$; block 2 rotated: $\mathrm{M}=18.5^{\circ}, \mathrm{SD}=7.89^{\circ}$; block 3 rotated: $\mathrm{M}=30.0^{\circ}, \mathrm{SD}=6.26^{\circ}$; block 4 rotated: $\left.\mathrm{M}=33.7^{\circ}, \mathrm{SD}=4.58^{\circ} ; p<0.05\right)$. At time $2, \mathrm{PVAE}$ did not differ between block 3 rotated and block 4 rotated $(p=1.00)$. However, at time 2 , block 2 rotated, participants had lower PVAE from all other rotated blocks (time 2: block $2: \mathrm{M}=31.9^{\circ}, \mathrm{SD}=1.73^{\circ}$; block $3: \mathrm{M}=35.1^{\circ}, \mathrm{SD}=$ $1.82^{\circ}$; block $\left.4: \mathrm{M}=35.9^{\circ}, \mathrm{SD}=1.87^{\circ}, p<0.05\right)$. Finally, while ANOVA revealed a group x time interaction, post hoc analysis indicated that all groups were reaching with similar errors at the start and end of each of the rotated blocks (all $p>0.05$ ). Thus, by the end of each rotated block, all participants reached to the target with similar errors at PVAE, regardless of group.

\section{Reaction time (RT) and Movement time (MT)}

In terms of RT, ANOVA revealed no differences between groups $(F(2,40)=721.710, p=$ $.864)$, or across blocks $(F(3,40)=1.288, p=0.282)$. Participants did exhibit shorter RT at time 2 compared to time 1 (i.e., shorter RT for trials completed at the end of each of the blocks compared to at the beginning; $F(1,40)=18.530, p<0.001$; time $1: \mathrm{M}=269.5 \mathrm{~ms}, \mathrm{SD}=17.09 \mathrm{~ms}$; time 2: $\mathrm{M}=233.2 \mathrm{~ms}, \mathrm{SD}=30.33 \mathrm{~ms})$.

With respect to MT, ANOVA reveled main effects of group $(F(2,40)=1294.265, p=$ $0.021)$, block $(F(3,40)=41.620, p<0.001)$, and time $(F(1,40)=64.018, p<0.001)$, as well as a 
Running Head: EXPLICIT AND IMPLICIT VISUOMOTOR ADAPTATION

significant block x time interaction $(F(3,40)=19.765, p<0.001)$. Overall, participants in the VRF No-Cursor group reached with the longest MT $(M=1130.6 \mathrm{~ms}, \mathrm{SD}=260.3 \mathrm{~ms})$, which was significantly greater than the MT observed for participants in the VRF group $(p=.025 ; \mathrm{M}=936.9$ $\mathrm{ms}, \mathrm{SD}=253.3 \mathrm{~ms})$. Participants in the PDP group reached with a mean MT of $966.4 \mathrm{~ms}(\mathrm{SD}=$ $254.9 \mathrm{~ms}$ ), which did not differ from either group (both $p>0.077$ ). Participants reached with the shortest MT in block 1 aligned $(M=880.4 \mathrm{~ms}, \mathrm{SD}=248.4 \mathrm{~ms})$, which did not change within the block (i.e., MT at time 1 was similar to time 2). MT increased with the introduction of the $40^{\circ} \mathrm{CW}$ visuomotor rotation in block 2 rotated $(\mathrm{M}=1152.34 \mathrm{~ms}, \mathrm{SD}=293.0 \mathrm{~ms})$, before decreasing significantly in block 3 rotated $(p<0.001 ; \mathrm{M}=1010.2 \mathrm{~ms}, \mathrm{SD}=229.7 \mathrm{~ms})$. ANOVA revealed a block $\mathrm{x}$ time interaction, and post hoc analysis revealed that participants had longer MT at time 1 than time 2 in all rotated blocks ( $<<0.001)$. Participants' MT was not different at the end of each rotated block (all $p>0.05$; block $2: \mathrm{M}=1001.1, \mathrm{SD}=250.1 \mathrm{~ms}$; block $3: \mathrm{M}=964.0 \mathrm{~ms}, \mathrm{SD}=$ $228.8 \mathrm{~ms}$; block 4: $\mathrm{M}=959.7 \mathrm{~ms}, \mathrm{SD}=204.5 \mathrm{~ms}$ ).

\section{Assessment Trials}

Explicit (EA) and Implicit Adaptation (IA)

Figure 5a shows the magnitude of EA established after each of the rotated blocks relative to errors in block 1 aligned for the PDP, VRF and VRF No-Cursor groups. Importantly, ANOVA revealed no main effect of group $(p=0.781)$, or any significant interactions involving group (all $p$ $>0.058)$. ANOVA did reveal a main effect of block $(F(2,40)=192.53, p=0.009)$, and post hoc analyses showed that the magnitude of EA increased slightly from block 2 rotated $\left(\mathrm{M}=18.44^{\circ}\right.$, $\left.\mathrm{SD}=11.86^{\circ}\right)$, to block 3 rotated $\left(p=0.022 ; \mathrm{M}=20.89^{\circ}, \mathrm{SD}=10.33^{\circ}\right)$ to block 4 rotated $(p=$ $0.042 ; \mathrm{M}=21.2^{\circ}, \mathrm{SD}=10.42^{\circ}$.

IA following each of the rotated blocks is shown in Figure $5 \mathrm{~b}$ for the 3 groups. ANOVA revealed a significant main effect of group $(F(2,40)=4.451, p=0.018)$, and time $(F(1,40)=$ 
Running Head: EXPLICIT AND IMPLICIT VISUOMOTOR ADAPTATION

$11.241, p=0.002)$. Post hoc analyses revealed that IA was greater in the VRF group $\left(M=13.51^{\circ}\right.$, $\left.\mathrm{SD}=1.27^{\circ}\right)$ compared to the VRF No-Cursor group $\left(p=0.015 ; \mathrm{M}=5.96^{\circ}, \mathrm{SD}=2.63^{\circ}\right)$. IA in the PDP group $\left(\mathrm{M}=9.61^{\circ}, \mathrm{SD}=5.87^{\circ}\right)$, did not differ from either group (both $\left.p \mathrm{~s}>0.332\right)$. Also, IA decayed from time $1\left(\mathrm{M}=10.9^{\circ}, \mathrm{SD}=2.80^{\circ}\right)$ to time $2\left(\mathrm{M}=8.47^{\circ}, \mathrm{SD}=3.00^{\circ}\right)$, regardless of group and block.

\section{Reaction time (RT) and Movement time (MT) in assessment trials}

In terms of RT in the VRF and VRF No-Cursor groups' assessment trials, ANOVA revealed main effects of block $(F(3,26)=4.445, p=0.006]$, and time $(F(1,26)=17.520, p<$ $0.001)$, and a significant interaction between block x time $(F(3,26)=3.478, p=0.040)$. Post hoc analysis failed to reveal a significant difference between blocks. RT was longer at time 1 compared to time $2(p<0.001$; time $1: \mathrm{M}=283.5 \mathrm{~ms}, \mathrm{SD}=82.13 \mathrm{~ms}$; time $2: \mathrm{M}=239.5 \mathrm{~ms}, \mathrm{SD}=45.51 \mathrm{~ms})$. In terms of MT for the VRF trials, ANOVA revealed main effects of group $(F(1,26)=4.940, p=$ $0.035)$ and block $(F(3,26)=7.068, p=0.002)$. Post hoc analysis revealed that the VRF No-Cursor group reached with a significantly longer $\mathrm{MT}(\mathrm{M}=1183.5 \mathrm{~ms}, \mathrm{SD}=270.7 \mathrm{~ms})$ compared to the VRF group $(p=0.035 ; \mathrm{M}=975.4 \mathrm{~ms}, \mathrm{SD}=246.5 \mathrm{~ms})$. Also, $\mathrm{MT}$ in block 2 rotated $(\mathrm{M}=1154$ $\mathrm{ms}, \mathrm{SD}=288.6 \mathrm{~ms}$ ) was significantly longer than the other three blocks (all $p \mathrm{~s}<0.033$; block 1 aligned: $\mathrm{M}=1017 \mathrm{~ms}$; block 3 rotated: $\mathrm{M}=1085 \mathrm{~ms}$; block 4 rotated: $\mathrm{M}=1062 \mathrm{~ms}$ )

For the PDP group, ANOVA revealed a main effect of block $(F(3,28)=3.282, p=0.025)$ with respect to RT when comparing performance across the exclusion and inclusion trials. While there was a trend of decreasing RT across blocks, post hoc analyses revealed no significant differences between blocks (all $p>0.097$ ). Analysis of MT also revealed a main effect of block $(F(3,28)=4.357, p=0.007)$, such that $\mathrm{MT}$ in block 2 rotated $(\mathrm{M}=892 \mathrm{~ms}, \mathrm{SD}=233.2 \mathrm{~ms})$ was 
Running Head: EXPLICIT AND IMPLICIT VISUOMOTOR ADAPTATION

significantly longer than block 1 aligned $(p=0.014 ; \mathrm{M}=813 \mathrm{~ms}, \mathrm{SD}=232.6 \mathrm{~ms})$. Overall, there was no difference in RT and MT between exclusion and inclusion trials in the PDP group.

The contributions of EA and IA to visuomotor adaptation

Figure 6 shows the percentage of reach adaptation which can be accounted for by the extent of EA + IA observed in each rotated block. ANOVA revealed a significant main effect of group $(F(2,40)=5.497, p=0.008)$, time $(F(1,40)=9.782, p=0.003)$, and significant interactions between group $\mathrm{x}$ block $(F(3.448,68.965)=3.858, p=0.010)$, group $\mathrm{x}$ time $(F(2,40)=4.557, p=$ 0.016), and group $\mathrm{x}$ block $\mathrm{x}$ time $(F(3.141,62.819)=2.950, p=0.037)$. Post hoc analysis revealed that the VRF group had a greater percentage of reach adaptation that was accounted for by EA and IA $(M=109 \%, S D=6.8 \%)$, compared to the VRF No-Cursor group $(\mathrm{M}=77 \%, \mathrm{SD}=32 \%)$ for all three of the rotated blocks at both times 1 and 2. The PDP groups' percentage of reach adaptation accounted for by EA and IA was on average 92\% (SD $=35 \%)$. This percentage did not differ from either that seen in the VRF or VRF No-Cursor groups, except in block 2 rotated, time 2, when it was less than that observed in the VRF group ( $p=.04 ; \mathrm{PDP}: \mathrm{M}=85.9 \%$; $\mathrm{SD}=46.8 \%$; VRF: $\mathrm{M}=121.6 \%, \mathrm{SD}=27.6 \%$ ). In general, the percentage of reach adaptation was significantly higher at time $1(\mathrm{M}=95 \%, \mathrm{SD}=28 \%)$ than time $2(\mathrm{M}=89 \%, \mathrm{SD}=34 \%)$ for all groups.

\section{Relationship between Explicit measures}

Figure 7a shows the relationship between verbal responses on the PEQ and EA in block 4 rotated, at time 1. Pearson correlation analyses revealed that the relationship between the PEQ and EA was significant for the PDP $\left(\mathrm{y}=0.7664 \mathrm{x}-14.951, R^{2}=.642 ; p<0.001\right)$, and VRF No-Cursor groups $\left(\mathrm{y}=0.9043 \mathrm{x}+10.303 ; R^{2}=0.4663, p=0.009\right)$, but not for the VRF group $(\mathrm{y}=0.3239 \mathrm{x}+$ $\left.23.139, R^{2}=0.0955, p=0.244\right)$. Figure $7 \mathrm{~b}$ shows the relationship between the errors drawn in the DT and EA in block 4 rotated, time 1. Again, Pearson correlation analyses revealed that the 
Running Head: EXPLICIT AND IMPLICIT VISUOMOTOR ADAPTATION

relationship between DT and EA was significant for the PDP $\left(\mathrm{y}=1.2414 \mathrm{x}-5.9184 ; R^{2}=0.7184\right.$, $p<0.001)$ and VRF No-Cursor groups $\left(\mathrm{y}=0.743 \mathrm{x}+7.8081 ; R^{2}=0.8164, p<0.001\right)$, but not for the VRF group $\left(\mathrm{y}=0.1265 \mathrm{x}+21.922 ; R^{2}=0.0713, p<0.317\right)$.

\section{Discussion}

In this study, we compared the contribution of explicit (Explicit Adaptation, EA: conscious, strategic changes in reaches) and implicit processes (Implicit Adaptation, IA: unconscious processes that arise due to sensory prediction errors) to visuomotor adaptation over time as assessed by different evaluation methods, in order to establish the relationship between explicit processes as assessed via Process Dissociation Procedure (PDP) and Verbal Report Framework (VRF). Three groups of participants adapted to a $40^{\circ} \mathrm{CW}$ visuomotor rotation in a similar manner. EA and IA were then assessed across blocks of trials using the PDP, VRF or VRF when no visual feedback was present (VRF No-Cursor group). Within the PDP assessment trials, participants reached without visual feedback under exclusion ('reach to the target') and inclusion ('reach using a strategy to get the cursor to the target') instructions. In the VRF assessment trials, participants reported a number indicating their planned aiming direction prior to reaching to the target. Awareness of the visuomotor rotation and changes in reaches were also assessed at the end of the experiment using a post-experiment questionnaire (PEQ) where participants were asked whether or not they noticed a change during training trials and a drawing task (DT) where participants were asked to draw the trajectory their hand took to get the cursor to the target in the training trials using a pen and paper.

\section{Explicit Adaptation (EA)}

Across the literature, a number of methods have been put forth to establish the role of explicit and implicit processes in visuomotor adaptation. These methods include the use of 
Running Head: EXPLICIT AND IMPLICIT VISUOMOTOR ADAPTATION

questionnaires (Benson et al., 2011; Modchalingam et al., 2019), drawing tasks (Larssen et al., 2012; Ong et al., 2012), eye gaze behaviour (de Brouwer, Albaghdadi, Flanagan, \& Gallivan, 2018), the PDP (Werner et al., 2015), and the VRF (Taylor, Krakauer, \& Ivry, 2014). Recently, the PDP and VRF assessments have received considerable attention. The PDP and VRF enable researchers to simultaneously assess both explicit and implicit contributions to visuomotor adaptation, regardless of participants' (verbally) reported level of awareness of the visuomotor distortion at the end of the experiment (Taylor et al., 2014; Bond \& Taylor, 20115; Werner et al., 2015; Neville \& Cressman, 2018; Modchalingam et al., 2019).

In the current experiment we compared EA as established via PDP and VRF assessments. We found that the magnitude of EA was similar across all three groups of participants following all rotated reach training blocks. EA increased slightly across blocks, peaking at $21.2^{\circ}$ in Block 4 . A similar increase in EA over training has been demonstrated by Bond and Taylor (2015), where EA increased in magnitude across reach training trials when participants trained to reach to two or four targets. In contrast, Taylor et al. (2014) have shown that EA decays across rotated reach training trials, when EA is assessed on a trial-by-trial basis during initial reach training trials and the contribution of IA increases. Here we assessed EA after 45 trials of rotated reach training. In accordance with the increase in EA, we also found an increase of $10^{\circ}$ in the extent of reach adaptation across rotated reach training blocks. Finally, we found that EA was retained across a delay of 5-minutes for all groups. These results extend recent findings from our lab, in which Bouchard and Cressman (2017) found that EA can be retained for up to 24-hours after rotated reach training trials, when assessed via the PDP. Specifically, we find that the magnitude and persistency of EA are similar regardless of method of assessment, when compared within the same experiment set-up, at similar times, and with a similar number of trials. 
Running Head: EXPLICIT AND IMPLICIT VISUOMOTOR ADAPTATION

While we have established similar patterns of EA across the PDP and VRF assessment paradigms with respect to magnitude and retention, it is unclear if they are assessing the same explicit processes. Differences between the PDP and VRF methods with respect to their definition and assessment of EA are subtle. Studies employing the PDP define EA as reflecting one's awareness of how to reach within the visuomotor environment (i.e., strategic reaching; Werner et al., 2015, 2019), while research using the VRF method has defined EA as reflecting pre-planned cognitive aiming strategies that participants plan to use to counteract the visuomotor rotation (Bond \& Taylor, 2015; Taylor et al., 2014). These aiming strategies are provided via verbal reports prior to reaching, and do not necessarily reflect awareness of the visuomotor distortion. That said, Morehead and colleagues (2015) have indicated that the processes underlying aiming strategies might be accessible to awareness.

In addition to the differences with respect to definition, EA is indirectly assessed within the PDP by calculating the difference between reaching errors in the exclusion versus the inclusion trials. In contrast, EA is directly assessed through verbal reports provided prior to reaching in the VRF. Values of EA correspond to a number's location in a number array surrounding the target in the VRF. By design, these numbers are separated by a minimal distance (e.g., 5.6 ${ }^{\circ}$; Taylor et al, 2014), thus placing constraints on the amount of EA that can be observed (i.e., it is not a continuous measure). In our VRF assessment trials, we attempted to limit the separation between our number locations to $3^{\circ}$. Even with this smaller separation between numbers, the measure of EA in the VRF is restricted to a value assigned to a number in the array surrounding the target, which is not the case in the PDP. These subtle differences in definition and assessment procedures make it unclear if the two methods are measuring similar explicit processes, even with the common pattern of results in the current experiment. 
Running Head: EXPLICIT AND IMPLICIT VISUOMOTOR ADAPTATION

In attempt to examine the explicit processes being assessed within the PDP and VRF in further detail, we looked to our responses on the post-experiment questionnaire (PEQ; from Benson et al., 2011; Hwang et al., 2006) and drawing task (DT; adapted from Larssen et al., 2012; Ong et al., 2012). Specifically, we looked to determine if EA as assessed via the PDP or VRF were related to participants' reported (verbal) awareness of the visuomotor distortion and how their reaches changed at the end of the experiment. In the PEQ, participants were questioned on whether they noticed a change during the experiment. Following a 'yes' answer, they were designated as aware of the visuomotor distortion and then probed on how they would describe that change, and finally, participants were asked to guess the size of the visuomotor rotation (see Benson et al., 2011 for PEQ). Within the DT participants were to draw the trajectory their hand took in order for the cursor to go to the target, while having vision of their hand. The task is adapted from Larssen et al. (2012), who suggest that the errors observed in the DT represent participants' awareness of the visuomotor distortion (2012). Based on our screening criteria, all participants were designated as aware of the visuomotor distortion based on their PEQ responses, Moreover, across groups, we found that average responses were similar on both the PEQ $\left(30.2^{\circ}\right)$ and DT $\left(22.8^{\circ}\right)$.

While group performance across PDP and VRF methods of assessment, as well as performance on the PEQ and DT, were similar with respect to explicit contributions to visuomotor adaptation, analyses of participants' individual performance data revealed a different story. In particular, we found that post-experiment awareness of the visuomotor distortion, as determined by the PEQ or DT, predicted EA only for the PDP and VRF-No Cursor groups. Within the PDP and VRF-No Cursor groups, slopes ranged from 0.6 to 1.1 (PDP: PEQ slope $=0.8377, \mathrm{R}^{2}=0.642$, DT slope $=0.5787, \mathrm{R}^{2}=0.718$; VRF-No Cursor: PEQ slope $=0.6255, \mathrm{R}^{2}=0.546, \mathrm{DT}$ slope $=$ $1.0988, \mathrm{R}^{2}=0.816$ ), such that participants who reported a greater visuomotor distortion in the PEQ 
Running Head: EXPLICIT AND IMPLICIT VISUOMOTOR ADAPTATION

and drew larger errors in the DT, demonstrated larger EA during assessment trials. On the other hand, there was no significant relationship between EA and responses on the PEQ and DT for the VRF group, even though their group performance was similar to that seen in the PDP and VRFNo Cursor groups. These results suggest that EA, as assessed via the PDP, is related to one's awareness of the visuomotor distortion and how one has to change their movements at the end of the experiment, while EA as assessed via the typical VRF assessment method does not reflect one's post-experiment awareness.

We modeled our VRF assessment trials off Taylor and colleagues (2014, other years), such that visual feedback of the cursor was presented during assessment of EA and IA for the VRF group. Participants first reported where they planned to reach in order to get to the cursor to the target, and then reached while seeing the cursor. The visual feedback enabled them to correct their movements online or on a trial-to-trial basis, and provided the opportunity to experience minimal errors (i.e., they saw the cursor go to the target). The PDP and VRF-No Cursor groups did not have the same opportunities with respect to correcting their movements or seeing a successful movement completed, as they did not see the cursor during any of the assessment trials. It is thus not surprising that a greater proportion of reach adaptation was accounted for by EA and IA in the VRF group ( 108\%) compared to the other two groups (PDP: 92\%; VRF-No Cursor: 77\%). The continued movement success of the VRF group may have led to a decreased awareness of the visuomotor distortion over the course of the experiment, as they would have experienced small target errors (i.e., the cursor went to the target).

Given these differences across groups, we conclude that the PDP and VRF do not assess similar explicit processes, and that the availability of visual feedback during assessment determines the role of awareness in EA. Specific to the PDP, EA reflects one's knowledge of how 
Running Head: EXPLICIT AND IMPLICIT VISUOMOTOR ADAPTATION

to reach to counteract visuomotor distortion. This EA is accessible to conscious awareness and can be recalled, such that participants can verbally report the magnitude of the visuomotor distortion and demonstrate how they had to change their movements at the end of the experiment. In the VRF method, EA reflects transient pre-planned aiming strategies that are contingent on the continued availability of visual feedback. These aiming strategies do not reflect participants' awareness of the visuomotor distortion at a later time.

\section{Implicit adaptation (IA)}

In order to determine if the PDP and VRF assess similar processes underlying visuomotor adaptation, we focussed on comparing EA across paradigms. However, within both the PDP and VRF, IA is also established. IA has been consistently defined within visuomotor adaptation paradigms, and across methods of assessment, as changes in reaches that arise due to unconscious and non-intentional changes to the internal model as a result of experiencing a sensory prediction error (Wolpert \& Kawato, 1998). Within the PDP and VRF, participants have a goal target and IA is established based on errors relative to the target. Given the consistency in definition of IA and similarities in method of assessment, it is perhaps not that surprising that we found similar IA between PDP and VRF assessments with respect to magnitude across reach training blocks and retention. We found that IA was on average $10.9^{\circ}$ at time 1 across the three rotated reach training blocks. For both the PDP and VRF groups, IA decayed by approximately $3^{\circ}$ following a 5 -minute break. This decay in IA has been well documented in visuomotor adaptation studies (see Morehead et al., 2015; Maksimovic \& Cressman, 2018; Nurouzpour et al., 2015). Specifically, previous work from our lab has shown that initial aftereffects decay by $71 \%$ following a 24 -hour period (i.e., from $24^{\circ}$ to $7^{\circ}$; Maksimovic \& Cressman, 2018). As well, an experiment using the PDP method reported 
Running Head: EXPLICIT AND IMPLICIT VISUOMOTOR ADAPTATION

evidence of the same decay of $\sim 3^{\circ}$ of IA following a 5-minute delay (Neville \& Cressman, 2018), as we see in the current experiment. These results indicate that IA is much less stable than EA.

We did find a difference in IA when we compared our VRF and VRF-No Cursor groups, such that the VRF No-Cursor group demonstrated less IA compared to the VRF group $\left(\sim 6.0^{\circ}\right.$ vs $14^{\circ}$, respectively). Within VRF assessment trials, IA was determined by the difference in reaching direction compared to the reported aiming direction. While visual feedback was manipulated between our VRF (cursor present) and VRF-No Cursor (cursor not present) groups, the number array was available throughout assessment trials for both groups, providing a continuous goal target. Participants in the VRF-No Cursor group aimed closer to their reported aiming direction compared to the VRF group. Previous research examining the role of visual feedback in visuomotor adaptation has shown that participants who adapted to a visuomotor distortion while receiving continuous visual feedback demonstrated larger reach aftereffects (larger IA) than those who received terminal feedback (smaller IA) (Barkley, Salomonczyk, Cressman, \& Henriques, 2014; Salomonczyk, Cressman, \& Henriques, 2011). The increased IA in the VRF group may have been promoted by the continually visible cursor, and opportunity to reach to the target (i.e., see the cursor go to the target). Furthermore, this increased IA, may explain why EA in the VRF group did not reflect their post-experiment awareness of the visuomotor distortion and how they had to change their reaches, as seen in the PDP and VRF-No Cursor groups.

\section{Summary}

Both explicit (EA) and implicit processes (IA) underlie visuomotor adaptation. To date, methods of assessment have used the term explicit to refer to knowledge regarding changes in reaches when reaching with a visuomotor distortion (PDP) or pre-planned aiming strategies (VRF). In the current experiment we asked if these explicit processes are similar across methods of 


\section{Running Head: EXPLICIT AND IMPLICIT VISUOMOTOR ADAPTATION}

assessment. We found similar EA with respect to magnitude over blocks of reach training trials and retention when assessed via the PDP and VRF following visuomotor adaptation. However, EA assessed in the PDP was related to participants' awareness of the visuomotor distortion and changes in their reaches as established by a post-experiment questionnaire (PEQ; Benson et al. 2011) and drawing task (DT; Larssen et al., 2011), respectively. Similar relationships were seen in the VRF assessment, only when the paradigm was modified and visual feedback regarding one's reaching trajectories was not available during assessment. Thus, the preplanned aiming strategy used to assess EA within the typical VRF design, does not reflect participants' awareness of the visuomotor distortion at the end of the experiment, and hence is measuring different explicit processes compared to the PDP. 
Running Head: EXPLICIT AND IMPLICIT VISUOMOTOR ADAPTATION

\section{References}

Baraduc, P., \& Wolpert, D. M. (2002). Adaptation to a visuomotor shift depends on the starting posture. Journal of Neurophysiology, 88(2), 973-981. https://doi.org/10.1152/jn.2002.88.2.973

Barkley, V., Salomonczyk, D., Cressman, E. K., \& Henriques, D. Y. P. (2014). Reach adaptation and proprioceptive recalibration following terminal visual feedback of the hand. Frontiers in Human Neuroscience, 8(SEP), 1-11. https://doi.org/10.3389/fnhum.2014.00705

Benson, B. L., Anguera, J. A., \& Seidler, R. D. (2011). A spatial explicit strategy reduces error but interferes with sensorimotor adaptation. Journal of Neurophysiology, 105(6), 2843-2851. https://doi.org/10.1152/jn.00002.2011

Bond, K. M., \& Taylor, J. A. (2015). Flexible explicit but rigid implicit learning in a visuomotor adaptation task. Journal of Neurophysiology, 113(10), 3836-3849. https://doi.org/10.1152/jn.00009.2015

Bouchard, J M., \& Cressman, E. (2019). Bimanual transfer and retention of visuomotor adaptation is driven by explicit processes. Journal of Exercise, Movement, and Sports (JEMS), 51(1).

Buch, E. R., Young, S., \& Contreras-Vidal, J. L. (2003). Visuomotor adaptation in normal aging. Learning and Memory, 10(1), 55-63. https://doi.org/10.1101//m.50303

Cressman, E. K., \& Henriques, D. Y. P. (2009). Sensory Recalibration of Hand Position Following Visuomotor Adaptation. Journal of Neurophysiology, 102(6), 3505-3518. https://doi.org/10.1152/jn.00514.2009

de Brouwer, A. J., Albaghdadi, M., Flanagan, J. R., \& Gallivan, J. P. (2018). Using gaze behavior to parcellate the explicit and implicit contributions to visuomotor learning. Journal of Neurophysiology, 120(4), 1602-1615. https://doi.org/10.1152/jn.00113.2018

Ghahramani, Z., Wolpert, D. M., \& Jordan, M. I. (1996). Generalization to local remappings of the 


\section{Running Head: EXPLICIT AND IMPLICIT VISUOMOTOR ADAPTATION}

visuomotor coordinate transformation. The Journal of Neuroscience : The Official Journal of the Society for Neuroscience, 16(21), 7085-7096. Retrieved from

http://www.ncbi.nlm.nih.gov/pubmed/8824344

Henriques, D. Y. P., \& Cressman, E. K. (2012). Visuomotor adaptation and proprioceptive recalibration. Journal of Motor Behavior, 44(6), 435-444.

https://doi.org/10.1080/00222895.2012.659232

Huang, V. S., Haith, A., Mazzoni, P., \& Krakauer, J. W. (2011). Rethinking Motor Learning and Savings in Adaptation Paradigms: Model-Free Memory for Successful Actions Combines with Internal Models. Neuron, 70(4), 787-801.

https://doi.org/10.1016/j.neuron.2011.04.012

Hwang, E. J., Smith, M. A., \& Shadmehr, R. (2006). Dissociable effects of the implicit and explicit memory systems on learning control of reaching. Experimental Brain Research, 173(3), 425-437. https://doi.org/10.1007/s00221-006-0391-0

Krakauer, J W, Pine, Z. M., Ghilardi, M. F., \& Ghez, C. (2000). Learning of visuomotor transformations for vectorial planning of reaching trajectories. The Journal of Neuroscience : The Official Journal of the Society for Neuroscience, 20(23), 8916-8924. Retrieved from http://www.ncbi.nlm.nih.gov/pubmed/11102502

Krakauer, J W., Ghilardi, M.-F., Mentis, M., Barnes, A., Veytsman, M., Eidelberg, D., \& Ghez, C. (2004). Differential Cortical and Subcortical Activations in Learning Rotations and Gains for Reaching: A PET Study. Journal of Neurophysiology, 91(2), 924-933. https://doi.org/10.1152/jn.00675.2003

Krakauer, J W., Ghilardi, M. F., \& Ghez, C. (1999). Independent learning of internal models for kinematic and dynamic control of reaching. Nature Neuroscience, 2(11), 1026-1031. 


\section{Running Head: EXPLICIT AND IMPLICIT VISUOMOTOR ADAPTATION}

https://doi.org/10.1038/14826

Larssen, B. C., Ong, N. T., \& Hodges, N. J. (2012). Watch and learn: Seeing is better than doing when acquiring consecutive motor tasks. PLoS ONE, 7(6). https://doi.org/10.1371/journal.pone.0038938

McDougle, S. D., Bond, K. M., \& Taylor, J. A. (2015). Explicit and Implicit Processes Constitute the Fast and Slow Processes of Sensorimotor Learning. Journal of Neuroscience, 35(26), 9568-9579. https://doi.org/10.1523/jneurosci.5061-14.2015

Modchalingam, S., Vachon, C. M., Hart, B. M. t., \& Henriques, D. Y. P. (2019). The effects of awareness of the perturbation during motor adaptation on hand localization. PLoS ONE, 14(8), 1-20. https://doi.org/10.1371/journal.pone.0220884

Neville, K. M., \& Cressman, E. K. (2018). The influence of awareness on explicit and implicit contributions to visuomotor adaptation over time. Experimental Brain Research, 236(7), 2047-2059. https://doi.org/10.1007/s00221-018-5282-7

Oldfield. (1971). Hand Preference. Encyclopedia of Clinical Neuropsychology. https://doi.org/10.1007/978-0-387-79948-3_6053

Ong, N. T., \& Hodges, N. J. (2010). Absence of after-effects for observers after watching a visuomotor adaptation. Experimental Brain Research, 205(3), 325-334. https://doi.org/10.1007/s00221-010-2366-4

Ong, N. T., Larssen, B. C., \& Hodges, N. J. (2012). In the absence of physical practice, observation and imagery do not result in updating of internal models for aiming. Experimental Brain Research, 218(1), 9-19. https://doi.org/10.1007/s00221-011-2996-1

Poh, E., Carroll, T. J., \& Taylor, J. A. (2016). Effect of coordinate frame compatibility on the transfer of implicit and explicit learning across limbs. Journal of Neurophysiology, 116(3), 
Running Head: EXPLICIT AND IMPLICIT VISUOMOTOR ADAPTATION

1239-1249. https://doi.org/10.1152/jn.00410.2016

Salomonczyk, D., Cressman, E. K., \& Henriques, D. Y. P. (2011). Proprioceptive recalibration following prolonged training and increasing distortions in visuomotor adaptation.

Neuropsychologia, 49(11), 3053-3062.

https://doi.org/10.1016/j.neuropsychologia.2011.07.006

Taylor, J. A., \& Ivry, R. B. (2014). Cerebellar and Prefrontal Cortex Contributions to Adaptation, Strategies, and Reinforcement Learning. Progress in Brain Research (1st ed., Vol. 210).

Elsevier B.V. https://doi.org/10.1016/B978-0-444-63356-9.00009-1

Taylor, Krakauer, \& Ivry. (2014). Explicit and Implicit Contributions to Learning in a

Sensorimotor Adaptation Task. Journal of Neuroscience, 34(8), 3023-3032.

https://doi.org/10.1523/JNEUROSCI.3619-13.2014

Wang, J., \& Lei, Y. (2015). Direct-effects and after-effects of visuomotor adaptation with one arm on subsequent performance with the other arm. Journal of Neurophysiology, 114(1), 468473.

Werner, S., Strüder, H. K., \& Donchin, O. (2019). Intermanual transfer of visuomotor adaptation is related to awareness. PLoS ONE, 14(9), 1-19. https://doi.org/10.1371/journal.pone.0220748

Werner, S., Van Aken, B. C., Hulst, T., Frens, M. A., Van Der Geest, J. N., Strüder, H. K., \& Donchin, O. (2015). Awareness of sensorimotor adaptation to visual rotations of different size. PLoS ONE, 10(4), 1-18. https://doi.org/10.1371/journal.pone.0123321

Wolpert, D. M., \& Kawato, M. (1998). Multiple paired forward and inverse models for motor control. Neural Networks, 11(7-8), 1317-1329. https://doi.org/10.1016/S08936080(98)00066-5 


\section{Figures}
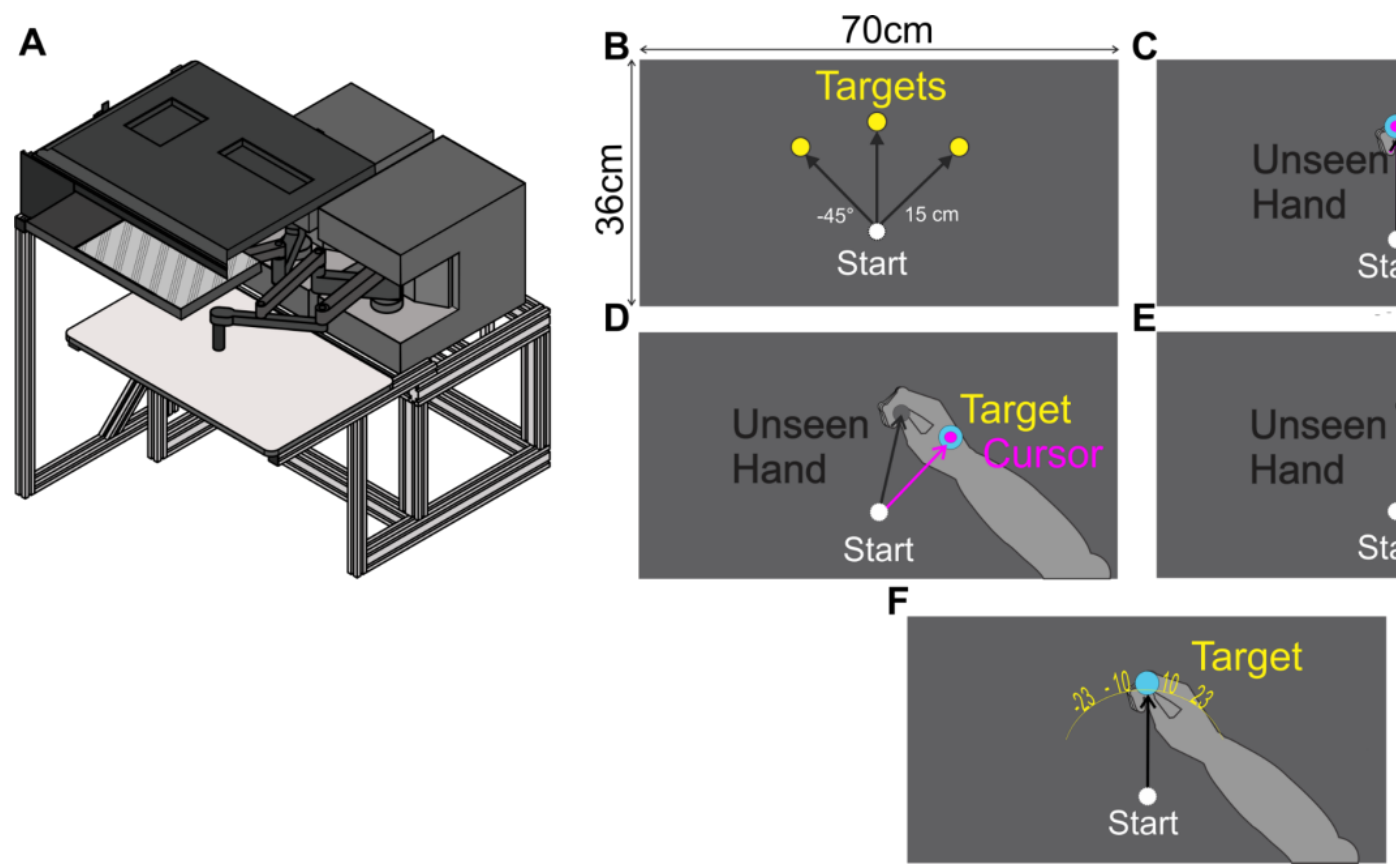

Figure 1. Experimental apparatus and types of trials

(A) Depiction of the KINARM: the double-jointed robot manipulandum used for data collection. Participants were seated on a height adjustable chair (not shown) and instructed to grasp the robot handle with their right hand. (B) Top view of the apparatus illustrating the dimensions of the experimental workspace. The home position (as represented by the white circle) was visible to participants throughout testing. The three reach targets (filled yellow circles, $2 \mathrm{~cm}$ in diameter), were located straight ahead, and $45^{\circ}$ clockwise $(\mathrm{CW})$ and $45^{\circ}$ counterclockwise $(\mathrm{CCW})$ relative to straight ahead, at a distance of $15 \mathrm{~cm}$ from the home position. (C) Top view of an aligned reach training trial. (D) Top view of a rotated reach training trial. (E) An example of an inclusion trial completed by the PDP group, where participants reached with no visual feedback of their hand position when asked to use any strategies they might have learned. (F) An example of an assessment trial completed by the VRF No-Cursor group, where numbered landmarks spanned $138^{\circ}$ around the target. 
Running Head: EXPLICIT AND IMPLICIT VISUOMOTOR ADAPTATION

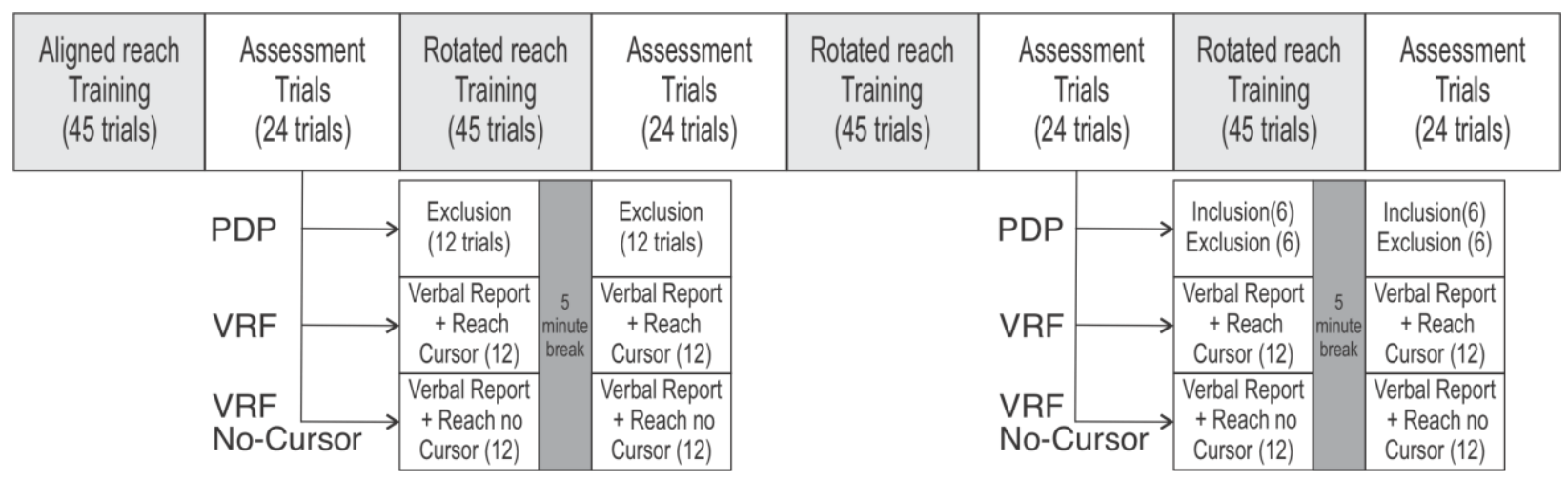

Figure 2. Experimental design

Overall breakdown of blocks completed by all participants and specific assessment blocks for participants in each of the PDP, VRF and VRF No-Cursor groups. In the PDP group, the order of exclusion and inclusion trials was counterbalanced across participants. In addition, the order of these trials was the same at time 1 (initial) and time 2 (5-minute delay), such that half of the participants started with exclusion trials, the other half started with inclusion trials. In the VRF and VRF No-Cursor groups, there was no difference between assessment trials after aligned reach training and rotated reach training. 
Running Head: EXPLICIT AND IMPLICIT VISUOMOTOR ADAPTATION

Home Position $\bigcirc$ Unseen Hand Position $\bigcirc$ Target Position $\bigcirc$ Cursor Position

A: Aligned Cursor reach training trial

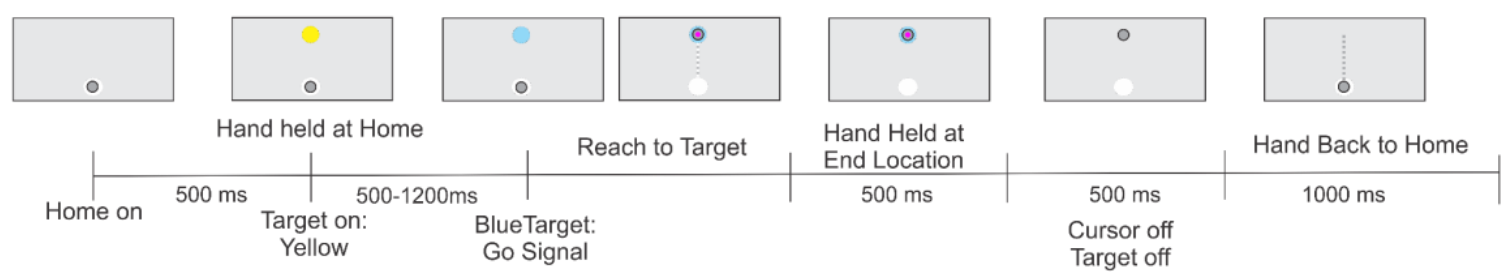

B: Rotated Cursor reach training trial
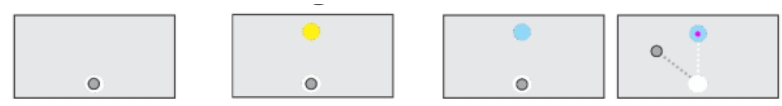

Hand held at Home

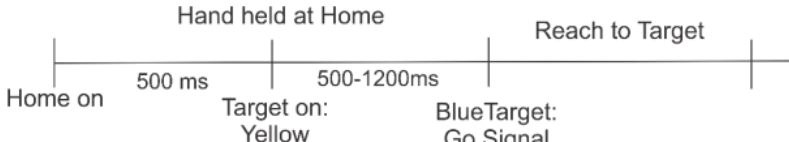

Yellow

Go Signal

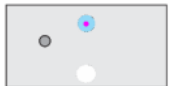

Hand Held at End Location $500 \mathrm{~ms}$

Inclusion Trials

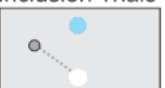

$\mathrm{C}$ : PDP: exclusion and inclusion trials
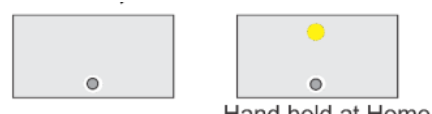

Hand held at Home
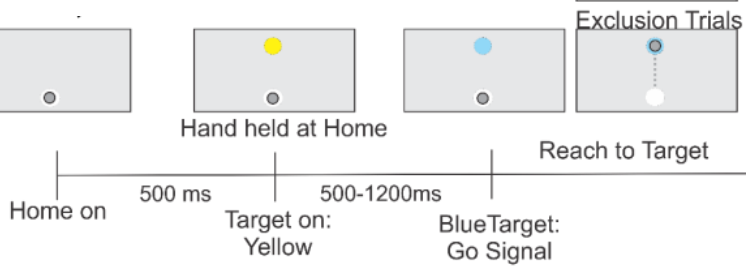

Reach to Target

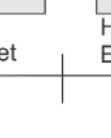

Hand Held at

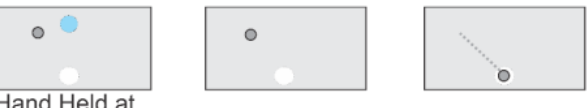

End Location

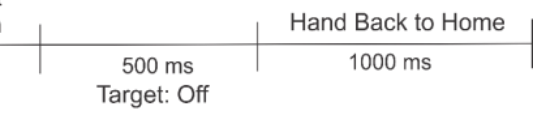

D: VRF: Verbal report trials

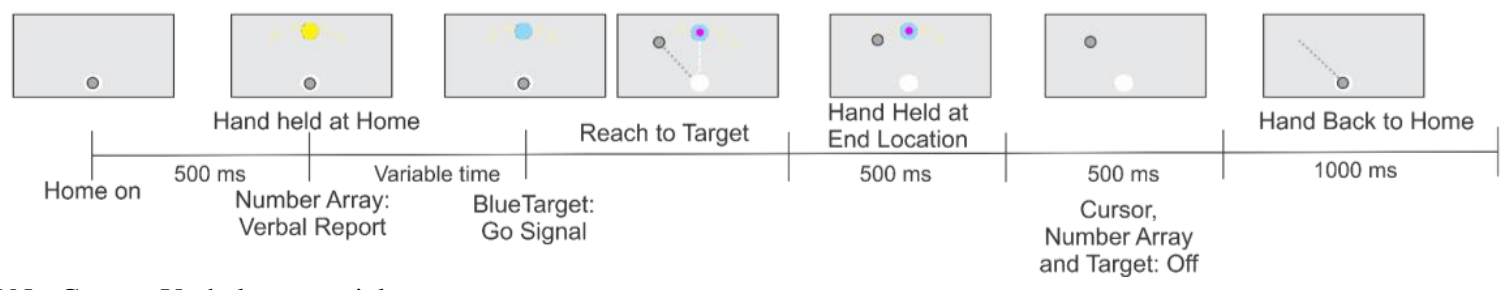

E: VRF No-Cursor: Verbal report trials

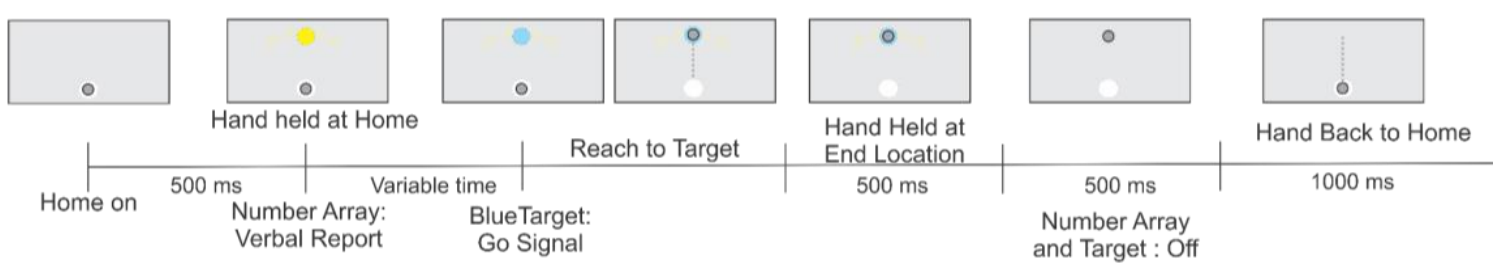

Figure 3. Sequence of events within each trial type

(A) Time and events in an aligned reach training trial and (B) a rotated reach training trial where the cursor position was rotated $40^{\circ} \mathrm{CW}$ relative to hand motion. (C) Time and events in the PDP exclusion and inclusion assessment trials. (D, E) VRF assessment trials where a number array was presented around the target. Participants first reported their planned aiming direction and then 


\section{Running Head: EXPLICIT AND IMPLICIT VISUOMOTOR ADAPTATION}

reached to the target while the cursor was available (D, VRF group), and unavailable (E, VRF NoCursor group). 
Running Head: EXPLICIT AND IMPLICIT VISUOMOTOR ADAPTATION

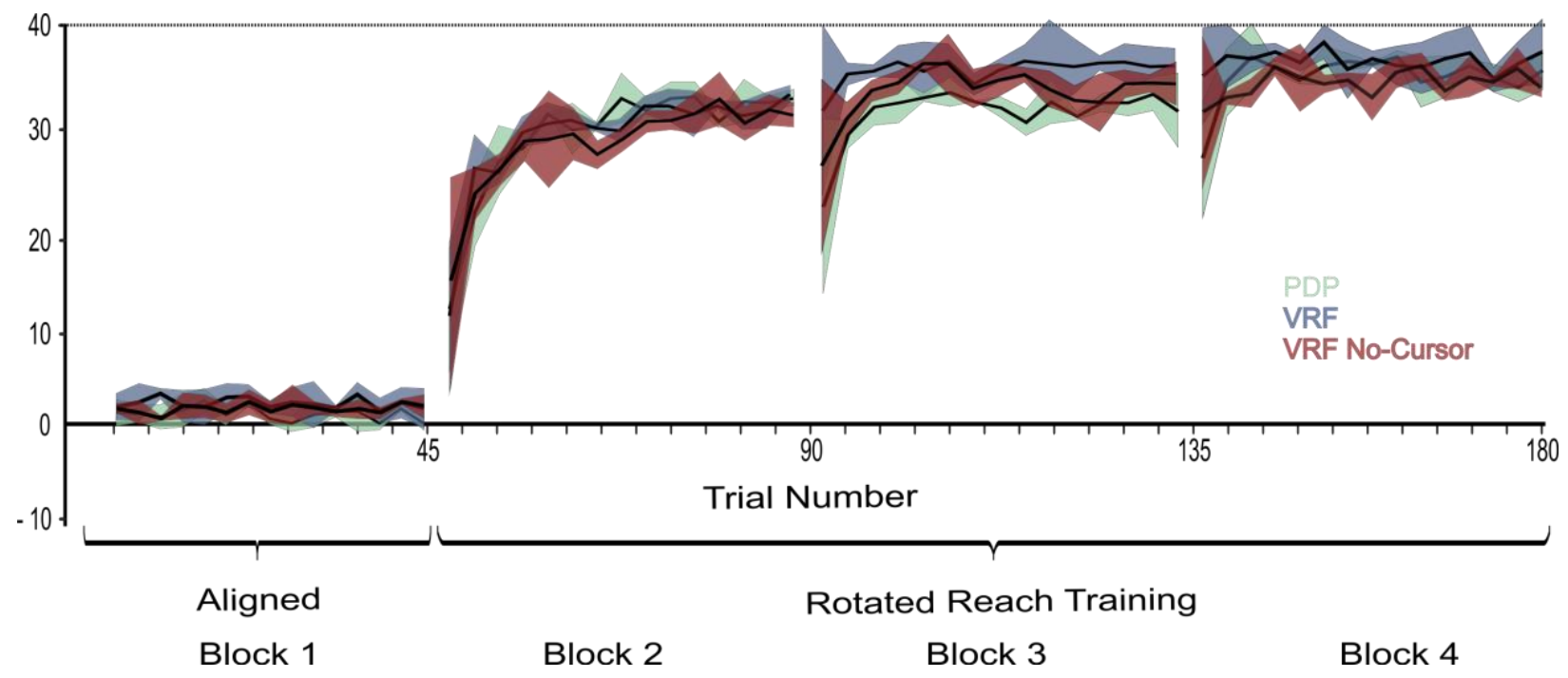

Figure 4. Reach Training Performance

Mean PVAE of the hand when participants trained with a cursor that was aligned (Block 1) or rotated $40^{\circ}$ clockwise $(\mathrm{CW})$ relative to their hand motion (Blocks 2, 3, 4). The results are presented such that each point represents average performance across three trials. Positive values indicate reaching to the left of the target. The PDP group is represented by the green area represented in green, the VRF group by the area represented in blue and the VRF No-cursor group by the area represented by red. The solid black lines represent mean reach performance, with the shaded region representing standard error of the mean in the positive and the negative directions. The dashed line represents full compensation for the $\mathrm{CW}$ rotation $\left(40^{\circ}\right)$. 
Running Head: EXPLICIT AND IMPLICIT VISUOMOTOR ADAPTATION
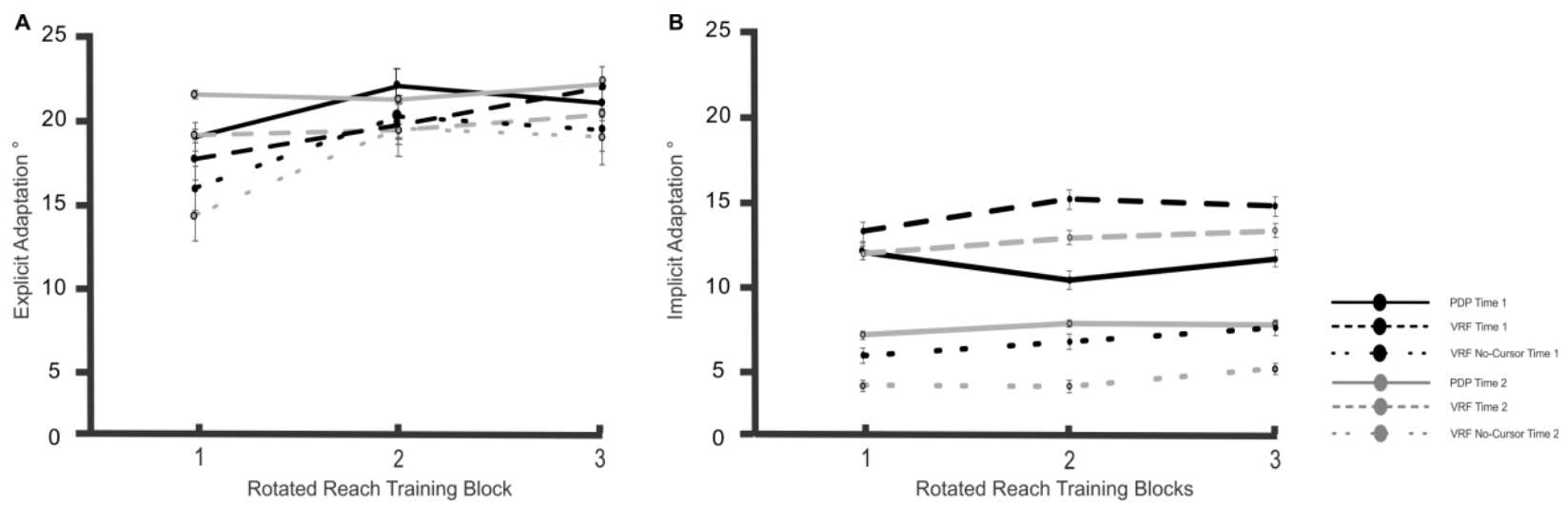

Figure 5. Explicit and Implicit adaptation

Mean Explicit Adaptation (A) and Implicit Adaptation (B) across each rotated reach training block at time 1 (Black lines) and time 2 (Grey lines). For each plot, solid lines represent the PDP group, large dashed lines represent the VRF group and the small dashed lines represent the VRF No-Cursor group. The error bars indicate standard error of the mean. 


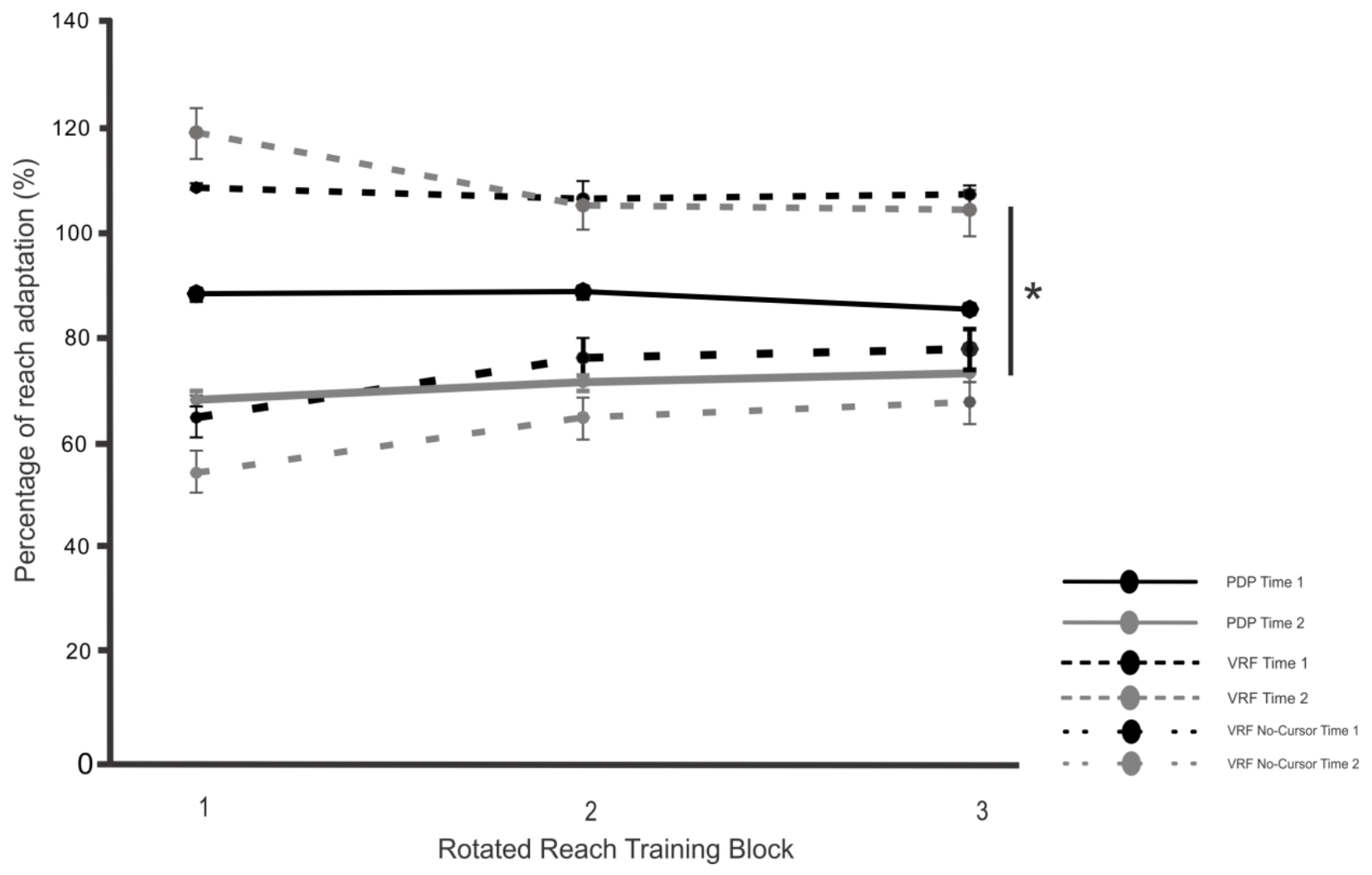

Figure 6. Adaptation indices as a percentage of reach adaptation

Explicit plus implicit adaptation expressed as a percentage of reach adaptation observed in each rotated block. Solid lines represent the PDP group, large dashed lines represent the VRF group and the small dashed lines represent the VRF No-Cursor group at time 1 (Black lines) and time 2 (Grey lines). The asterisk $(*)$ indicates a significant difference between the VRF and VRF No-Cursor groups. Error bars indicate the standard error of the mean. 

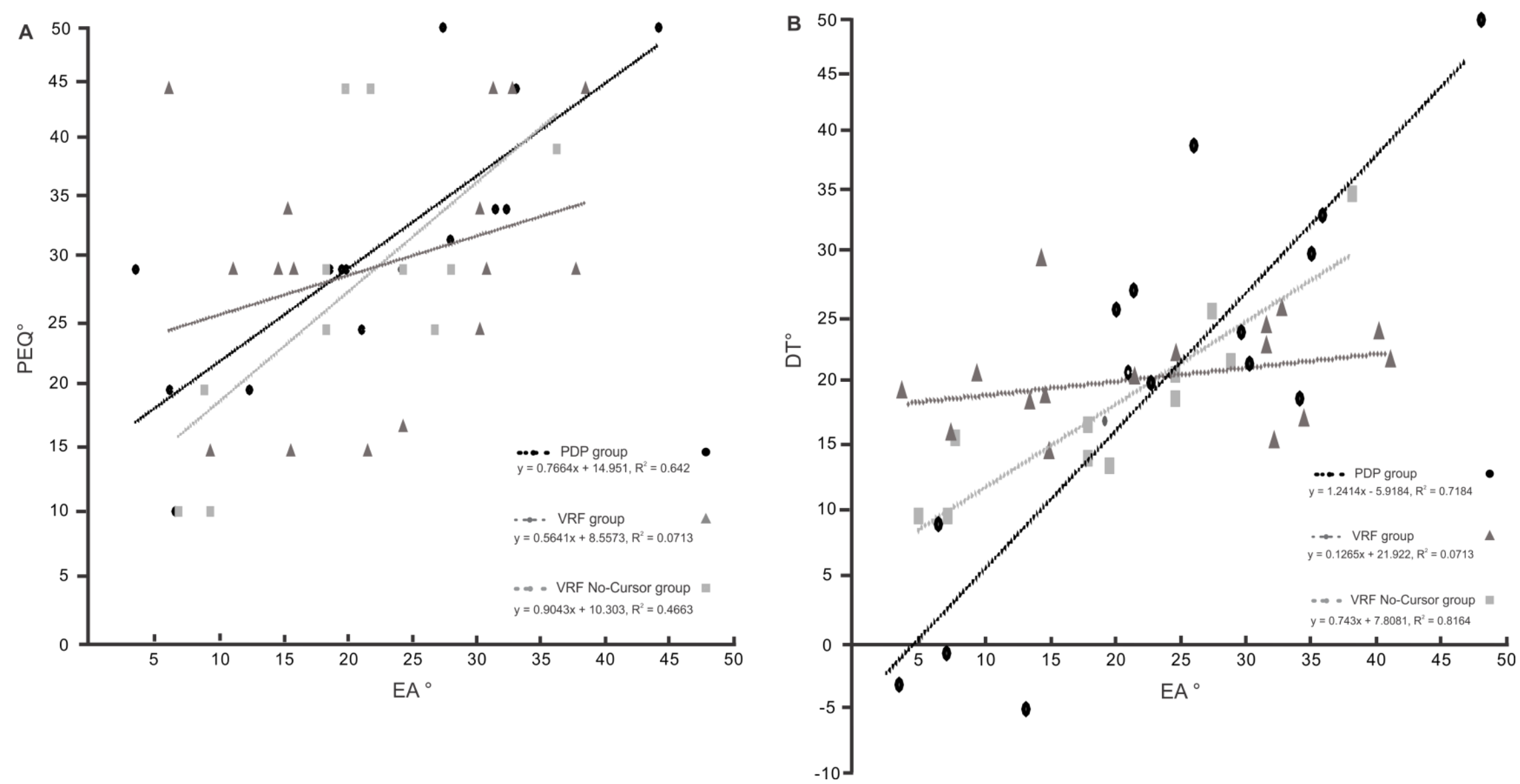

Figure 7. Relationship between methods of assessment

(A) The relationship between the magnitudes of EA as established in the assessment trials in rotated block 4, at time 1, and responses on the post-experiment questionnaire. (B) The relationship between the magnitude of EA in the assessment trials, in rotated block 4, time 1, and errors on the DT. Circles represent the PDP group, triangles represent the VRF group, and squares represent the VRF NoCursor group. 


\section{General Discussion}

Motor adaptation, a form of motor learning, can be examined by assessing changes in reaches in response to a visuomotor distortion. In the current experiment, all participants adapted their reaches to a $40^{\circ}$ visuomotor distortion. The main goal of our study was to compare the extent of explicit (EA) and implicit adaptation (IA) observed when assessed via the Process Dissociation Procedure (PDP) and Verbal Report Framework (VRF), in order to establish if the 2 methods of assessment assess similar processes underlying visuomotor adaptation. Further, given our interest in the contributions of EA to visuomotor adaptation and diversity of assessment methods used previously, we also included the post-experiment questionnaire (PEQ) and a Drawing task (DT) to establish the relationship between EA as assessed via the PD and VRF and participants' postexperiment awareness of the visuomotor distortion and changes in their reaches. Finally, we examined the role of visual feedback in VRF assessment trials by including a third group of participants (i.e., the VRF No-Cursor group), who completed the same assessment trials as the VRF group but did not receive visual feedback during the trials.

In general, we found no differences in the magnitude or retention of EA and IA between the PDP and VRF groups. IA did differ between the VRF and VRF No-Cursor groups, such that IA was less in the VRF No-Cursor group. For participants in the PDP and VRF No-Cursor groups, EA was related to their awareness of the visuomotor distortion (i.e., PEQ responses) and how they had to change their reaches (i.e. DT errors) at the end of the experiment. However, EA assessed in the VRF group was not related to their awareness of the magnitude of the visuomotor distortion at the end of the experiment nor how they had to change their reaches, as determined by their responses on the PEQ and DT, respectively. The increased IA in the VRF group may have been driven by access to continual visual feedback throughout the experiment, which would have 
Running Head: EXPLICIT AND IMPLICIT VISUOMOTOR ADAPTATION

provided information regarding reaching errors on a trial-to-trial basis, potentially reducing the role of awareness in changes to reaches over trials. Thus, we suggest that our findings indicate that the PDP and VRF methods do not assess similar explicit processes underlying visuomotor adaptation.

\section{Tests of Awareness}

We included the PEQ and DT to assess participants' awareness of the visuomotor distortion and changes in their reaches at the end of the experiment. The PEQ was adopted from Benson et al. (2011), and has been used extensively as a post-experiment assessment of awareness of the visuomotor distortion. As in our experiment, previous work has typically administered the questionnaire at the end of the experiment (e.g., Bouchard \& Cressman, 2019; Wang, Joshi \& Lei, 2011; Taylor, Wojaczynski, \& Ivry, 2011), at which time reaching errors tend to be quite small, potentially impacting participants' awareness of the visuomotor perturbation. Initial work using such questionnaires classified participants as aware or unaware of the visuomotor rotation (Benson et al., 2011; Hwang et al., 2006), and then regarded participants' performance as being driven explicitly (if aware of the visuomotor distortion) or implicitly (if unaware of the visuomotor distortion). Today, it has been suggested that categorizing participants as aware versus unaware and hence engaging explicit vs. implicit processes, is too strict. There is increasing evidence that both explicit and implicit processes can contribute to visuomotor adaptation, regardless of participants' reported awareness at the end of the experiment (Neville \& Cressman, 2018; Taylor et al., 2014; Werner et al., 2015). In the current experiment, we used the PEQ and DT to determine whether awareness at the end of the experiment was related to EA during visuomotor adaptation as established via PDP and VRF methods of assessment. Below we discuss the relationship between responses on the PEQ, DT and EA as assessed via the PDP and VRF. 
Running Head: EXPLICIT AND IMPLICIT VISUOMOTOR ADAPTATION

We found that participants' responses on the PEQ indicated that they perceived the visuomotor distortion as being approximately $30.2^{\circ}$. Interestingly, responses on the PEQ indicated greater awareness of the visuomotor distortion than errors drawn during the DT (approximately $23^{\circ}$ ), and indeed than the extent of EA established via the assessment trials (approximately $20^{\circ}$ ). This increased awareness on the PEQ is in contrast to previous suggestions that verbal reports tend to underestimate awareness due to differences in retrieval contexts or because knowledge is held in low confidence (Eriksen, 1960; Nisbett \& Wilson, 1977; Reingold \& Merikle, 1990; Shanks \& St John, 1994). That said, this suggestion is not based on visuomotor adaptation paradigms and to date, prior to our research, no study has compared participants' reported awareness following visuomotor adaptation with EA assessed during training trials.

In our full sample of participants $(\mathrm{N}=61)$, we had a number of individuals in each group that did not show evidence of EA in the PDP or VRF assessment trials but showed awareness of the visuomotor distortion at the end at the experiment as evaluated by their responses to the PEQ and DT (PDP: 2 participants, VRF: 0 participants; VRF-No Cursor: 1 participant). Alternatively, we also had a number of participants who did not show awareness of the visuomotor distortion at the end of the experiment using the PEQ and/or DT but demonstrated EA in the assessment trials (PDP: 1 participant, VRF: 4 participants; VRF-No Cursor: 1 participant). In an initial analysis, we included data from all 61 participants. Results from our full data set revealed similar patterns to those discussed in Chapter II; EA was similar with respect to magnitude and retention across our 3 groups of participants, but not related to post-experiment awareness in our VRF group. Given our question of interest, we decided to focus our research on participants who demonstrated EA and were aware of the distortion at the end of the experiment. That said, these 'discarded' participants, as well as differences in the relationship between EA and post-experiment awareness 
Running Head: EXPLICIT AND IMPLICIT VISUOMOTOR ADAPTATION

(see also Werner et al. (2015) and Modchalingam et al. (2019)), do raise questions regarding consistencies (or inconsistencies) across methods of assessment of explicit contributions to visuomotor adaptation. Below we suggest that differences across methods of assessment may be driven by the nature of the task (i.e., vision for perception vs. action).

\section{Perception versus Action}

The neural substrates underlying visual perception have been suggested to differ from those underlying the visual control of goal-directed actions (Milner \& Goodale, 1992). According to Milner and Goodale's dual model of visual processing (1992), the ventral (what) visual stream, projecting from primary visual cortex to the inferotemporal cortex, is responsible for object identification and perception of the visual world, whereas the dorsal (how) visual stream, projecting from primary visual cortex to the parietal cortex, is responsible for visually-guided actions (Goodale, Pelisson, \& Prablanc, 1986). Evidence supporting the two-stream model of visual processing has come from patient populations (Milner \& Goodale, 2006; Goodale, Milner, Jakobson, Carey, 1991; Jeannerod, 1986; Perening \& vighetto, 1988; 1983; Goodale et al., 1991; Jeannerod, Decety, \& Michel, 1994) and healthy individuals (Goodale et al., 1986; Desmurget et al., 1998). In the two-streams model of visual processing, perception refers to the conscious and unconscious experience of vision (Milner \& Goodale, 2008). Conscious experience of vision (i.e., visual experience of the visual stimuli) can be translated into subjective, verbal reports, while unconscious perception (i.e., mental representations of the visual stimuli that may not reach conscious awareness) does not translate into subjective reports. That said, unconscious perception can influence later cognitive operations in healthy participants (Dehaene et al., 1998).

In the PDP, EA and IA are established based on reaching errors (i.e., an action task), whereas in the VRF, EA is determined based on a verbal responses (i.e., a perceptual task), such 
Running Head: EXPLICIT AND IMPLICIT VISUOMOTOR ADAPTATION

that participants verbally indicate the number they plan to reach to prior to initiating their movement. Thus, given the different nature of the tasks employed by the PDP and VRF, perhaps they are tapping onto different visual processing streams when assessing EA. Moreover, in the VRF, IA is determined by both perceptual and motor responses, as IA reflects the difference between one's verbal report and their reaching errors. If perception can influence action, then does the measure of IA as established via the VRF method reflect similar processes as seen in the PDP?

In general, we found that EA and IA were similar in magnitude across the PDP and VRF methods. This similarity indicates that perhaps the two tasks were not that different. If we consider the PDP assessment trials in more detail, we find that participants were given instructions regarding their reaches. Specifically, the exclusion and inclusion instructions were to reach when you "using what you learned" and "not using what you learned", respectively. These instructions may have changed the nature of the action task, tapping into perceptual processing, as engaged in during the VRF assessment trials. Moreover, we delayed reach initiation for the PDP group, as they had to wait 500 to $1200 \mathrm{~ms}$ for the target to change from yellow to blue, before starting their movement. This delay may also have made the PDP assessment trials more similar to the VRF assessment trials, in which participants initiated their reaches following their verbal responses indicating where they planned to reach. Thus, perhaps the assessment trials for the PDP, which we refer to as a motor task, may have a perceptual component as well. In accordance with this suggestion we found similar RT and MT across exclusion and inclusion PDP trials, whereas in our previous work, in which we did not delay reach onset, we found that participants took longer to initiate their reaches in the inclusion trials compared to exclusion trials (Bouchard \& Cressman, 2019). This perceptual processing (i.e. movement planning) required by the PDP and VRF could 
Running Head: EXPLICIT AND IMPLICIT VISUOMOTOR ADAPTATION

explain the similar trends between the two methods. That said, the similarities between paradigms were contingent on visual feedback being available during the VRF assessment trials.

\section{Future directions}

In the current study, we compared EA as established via the PDP and VRF methods of assessment, at similar points of time, after participants completed the same number of reach training trials, to the same visuomotor distortion. In order to maintain participants' motivation (i.e., not make the experiment too long), participants completed a limited number of assessment trials. The PDP group completed 12 assessment trials, 6 exclusion and 6 inclusion trials, at each assessment time. The VRF groups also completed 12 assessment trials at each time, but all 12 trials provided an index of explicit and implicit processes. Perhaps this difference in trial number may have influenced the consistency of participants' responses across methods of assessment. Given the limited number of trials to each target, we did not asses the influence of target position, or consistency of reaches. Previous work from our lab (Neville \& Cressman, 2018), has demonstrated that explicit and implicit adaptation are similar across target positions when assessed using the PDP. Future research is needed to determine the relationship between consistency of EA and IA across methods of assessment, and determine if target position plays a role, as suggested by Day and colleagues (2016).

Future research could also look to determine the role of perceptual processes in establishing IA. For example, IA could be assessed within the VRF (and PDP), when explicit processes are not exploited. For example, participants could complete a series of catch trials during reach training trials, or a block of aftereffect trials (i.e., no-cursor reaches when instructed to reach to the target), when the number array is not presented around the target, to ensure that perceptual processes are not engaged. Results from these experiments would indicate if engaging perceptual processes (i.e., 
Running Head: EXPLICIT AND IMPLICIT VISUOMOTOR ADAPTATION

assessing EA), influence IA in the VRF. Similarly, participants could be instructed to merely reach to the target in the PDP assessment trials (i.e., employ standard instructions given in aftereffect trials), without the mention of "not using what you learned" or delaying reach onset. We have conducted preliminary work in our lab looking to study the influence of perceptual processes on IA (Decarie, Heirani Moghaddam \& Cressman, 2020). We tested 4 groups of subjects who adapted to a $40^{\circ}$ clockwise rotation and then IA was assessed using the PDP and VRF methods. Results indicated that the extent of IA was largest for the PDP group, when no instructions on strategy was provided. However, the magnitude of IA in the VRF group did not differ regardless of whether EA was assessed as well as IA or not. Taken together, the results indicated that the influence of assessing EA on IA depends on the method of assessment, such that assessing EA within the PDP framework leads to a decrease in the extent of IA observed. These results further support the idea that the PDP and VRF methods assess different processes.

\section{Conclusion}

Here we compared the magnitude and retention of explicit (EA) and implicit adaptation (IA) within the PDP and VRF methods. Additionally, we looked to establish the relationship between EA and awareness of the visuomotor distortion and changes in reaches at the end of the experiment. We found that the average magnitude and retention of EA and IA were similar regardless of method of assessment. However, the magnitude of EA observed in the VRF group was not related to their awareness of the visuomotor distortion or the changes in their reaches at the end of the experiment. Together, these results indicate that, while the PDP and VRF suggest similar contributions of EA and IA to visuomotor adaptation, the methods of assessment engage different explicit processes. EA assessed within the VRF does not reflect one's awareness of the visuomotor distortion at the end of the experiment or how they changed their reaches. 
Running Head: EXPLICIT AND IMPLICIT VISUOMOTOR ADAPTATION

\section{References}

Baraduc, P., \& Wolpert, D. M. (2002). Adaptation to a visuomotor shift depends on the starting posture. Journal of Neurophysiology, 88(2), 973-981.

Bastian, A. J. (2008). Understanding sensorimotor adaptation and learning for rehabilitation. Current Opinion in Neurology, 21(6), 628-633.

Benson, B. L., Anguera, J. A., \& Seidler, R. D. (2011). A spatial explicit strategy reduces error but interferes with sensorimotor adaptation. Journal of Neurophysiology, 105(6), 2843-2851. https://doi.org/10.1152/jn.00002.2011

Bergerbest, D., \& Goshen-Gottstein, Y. (2002). The origins of levels-of-processing effects in a conceptual test: Evidence for automatic influences of memory from the process-dissociation procedure. Memory \& cognition, 30(8), 1252-1262.

Bond, K. M., \& Taylor, J. A. (2015). Flexible explicit but rigid implicit learning in a visuomotor adaptation task. Journal of Neurophysiology, 113(10), 3836-3849. https://doi.org/10.1152/jn.00009.2015

Bouchard, J M., \& Cressman, E. (2019). Bimanual transfer and retention of visuomotor adaptation is driven by explicit processes. Journal of Exercise, Movement, and Sports (JEMS), 51(1).

Buch, E.R., Young, S., and Contreras-Vidal, J.L. (2003). Visuomotor adaptation in normal aging. Learning and Memory, 10, 55-63.

Cressman, E. K., \& Henriques, D. Y. (2009). Sensory recalibration of hand position following visuomotor adaptation. Journal of neurophysiology, 102(6), 3505-3518.

Day, K. A., Roemmich, R. T., Taylor, J. A., \& Bastian, A. J. (2016). Visuomotor learning generalizes around the intended movement. eneuro, 3(2).

Decarie, A., Heirani Moghaddam, S., Cressman, E. K., (2020). Assessing Explicit Adaptation Leads to a Decrease in Implicit Adaptation When Reaching in the Absence of Visual Cues. 
Running Head: EXPLICIT AND IMPLICIT VISUOMOTOR ADAPTATION

Perceptual Tasks Promote Explicit Adaptation. North American Society for the Psychology of Sport and Physical Activity (NASPSPA). Oral presentation.

Dehaene, S., Naccache, L., Le Clec'H, G., Koechlin, E., Mueller, M., Dehaene-Lambertz, G., ... \& Le Bihan, D. (1998). Imaging unconscious semantic priming. Nature, 395(6702), 597.

Desmurget, M., \& Grafton, S. (2000). Forward modeling allows feedback control for fast reaching movements. Trends in cognitive sciences, 4(11), 423-431.

Desmurget, M., Pelisson, D., Urquizar, C., Prablanc, C., Alexander, G. E., \& Grafton, S. T. (1998). Functional anatomy of saccadic adaptation in humans. Nature neuroscience, 1(6), 524.

Destrebecqz, A., \& Cleeremans, A. (2001). Can sequence learning be implicit? New evidence with the process dissociation procedure. Psychonomic bulletin \& review, 8(2), 343-350.

Destrebecqz, A., Peigneux, P., Laureys, S., Degueldre, C., Del Fiore, G., Aerts, J., ... \& Maquet, P. (2003). Cerebral correlates of explicit sequence learning. Cognitive Brain Research, 16(3), 391-398.

Eriksen, C. W. (1960). Discrimination and learning without awareness: A methodological survey and evaluation. Psychological Review, 67(5), 279-300. https://doi.org/10.1037/h0041622

Francis, B. A., \& Wonham, W. M. (1976). The internal model principle of control theory. Automatica, 12, 457-465.

Ghahramani, Z., Wolpert, D. M., \& Jordan, M. I. (1996). Generalization to local remappings of the visuomotor coordinate transformation. The Journal of Neuroscience : The Official Journal of the Society for Neuroscience, 16(21), 7085-7096. Retrieved from http://www.ncbi.nlm.nih.gov/pubmed/8824344

Glover, S. (2004). Separate visual representations in the planning and control of action. Behavioral and Brain Sciences, 27(01), 3-78. https://doi.org/10.1017/s0140525x04000020 
Running Head: EXPLICIT AND IMPLICIT VISUOMOTOR ADAPTATION

Goodale MA, Milner AD, Jakobson LS, Carey DP (1991) Perceiving the world and grasping it. A neurological dissociation. Nature 349:154-156.

Goodale, M. A., \& Milner, A. D. (1992). Separate visual pathways for perception and action. Trends in neurosciences, 15(1), 20-25.

Goodale, M. A., Pelisson, D., \& Prablanc, C. (1986). Large adjustments in visually guided reaching do not depend on vision of the hand or perception of target displacement. Nature, 320(6064), 748-750. https://doi.org/10.1038/320748a0

Goodale, M. A., Pelisson, D., \& Prablanc, C. (1986). Large adjustments in visually guided reaching do not depend on vision of the hand or perception of target displacement. Nature, 320(6064), 748.

Harris, C.S. (1963). Adaptation to displaced vision: Visual, motor, or proprioceptive change? Science, $140(1), 812-813$.

Henriques, D. Y. P., \& Cressman, E. K. (2012). Visuomotor adaptation and proprioceptive recalibration. Journal of Motor Behavior, 44(6), 435-444. https://doi.org/10.1080/00222895.2012.659232

Huang, V. S., Haith, A., Mazzoni, P., \& Krakauer, J. W. (2011). Rethinking Motor Learning and Savings in Adaptation Paradigms: Model-Free Memory for Successful Actions Combines with Internal Models. Neuron, 70(4), 787-801. https://doi.org/10.1016/j.neuron.2011.04.012

Hwang, E. J., Smith, M. A., \& Shadmehr, R. (2006). Dissociable effects of the implicit and explicit memory systems on learning control of reaching. Experimental Brain Research, 173(3), 425-437. https://doi.org/10.1007/s00221-006-0391-0

Jacoby, L. L. (1991). A process dissociation framework: Separating automatic from intentional uses 
Running Head: EXPLICIT AND IMPLICIT VISUOMOTOR ADAPTATION

of memory. Journal of memory and language, 30(5), 513-541.

Jeannerod, M. (1986). Mechanisms of visuomotor coordination: a study of normal and braindamaged subjects. Neuropsychologia 24:41-78

Jeannerod, M. (1988). The neural and behavioural organization of goal-directed movements (Vol. 15, pp. 228-244). Oxford: Clarendon Press.

Jeannerod, M., Decety, J., Michel, F., (1994). Impairment of grasping movements following a bilateral posterior parietal lesion. Neuropsychologia 32:369-380

Kagerer, F. A., Contreras-Vidal, J. L., \& Stelmach, G. E. (1997). Adaptation to gradual as compared with sudden visuo-motor distortions. Experimental Brain Research, 115(3), 557561. https://doi.org/10.1007/PL00005727

Kitazawa, S., Kimura, T., \& Uka, T. (1997). Prism adaptation of reaching movements: specificity for the velocity of reaching. Journal of Neuroscience, 17(4), 1481-1492.

Krakauer, J W, Pine, Z. M., Ghilardi, M. F., \& Ghez, C. (2000). Learning of visuomotor transformations for vectorial planning of reaching trajectories. The Journal of Neuroscience : The Official Journal of the Society for Neuroscience, 20(23), 8916-8924. Retrieved from http://www.ncbi.nlm.nih.gov/pubmed/11102502

Krakauer, J. W. (2009). Motor Learning and Consolidation: The Case of Visuomotor Rotation. Advances in Experimental Medicine and Biology, 629, 405-421.

Krakauer, J. W., Pine, Z. M., Ghilardi, M.F., \& Ghez, C. (2000). Learning of visuomotor transformations for vectorial planning of reaching trajectories. Journal of Neuroscience, 20(23), 8916-8924.

Krakauer, John W., Ghez, C., \& Ghilardi, M. F. (2005). Adaptation to visuomotor transformations: Consolidation, interference, and forgetting. Journal of Neuroscience, 25(2), 473-478. 


\section{Running Head: EXPLICIT AND IMPLICIT VISUOMOTOR ADAPTATION}

https://doi.org/10.1523/JNEUROSCI.4218-04.2005

Krakauer, John W., Ghilardi, M. F., \& Ghez, C. (1999). Independent learning of internal models for kinematic and dynamic control of reaching. Nature Neuroscience, 2(11), 1026-1031. https://doi.org/10.1038/14826

Larssen, B. C., Ong, N. T., \& Hodges, N. J. (2012). Watch and learn: Seeing is better than doing when acquiring consecutive motor tasks. PLOS ONE, 7(6). https://doi.org/10.1371/journal.pone.0038938

Maksimovic, \& Cressman. (2018). Long-term retention of proprioceptive recalibration. Neuropsychologia, 114, 65-76.

Martin, T. A., Keating, J. G., Goodkin, H. P., Bastian, A. J., \& Thach, W. T. (1996). Throwing while looking through prisms: I. Focal olivocerebellar lesions impair adaptation. Brain, 119(4), 1183-1198.

McDougle, S. D., Bond, K. M., \& Taylor, J. A. (2015). Explicit and Implicit Processes Constitute the Fast and Slow Processes of Sensorimotor Learning. Journal of Neuroscience, 35(26), 9568-9579. https://doi.org/10.1523/jneurosci.5061-14.2015

Milner, A. D., \& Goodale, M. A. (2008). Two visual systems re-viewed. Neuropsychologia, 46(3), 774-785. https://doi.org/10.1016/j.neuropsychologia.2007.10.005

Modchalingam, S., Vachon, C. M., Hart, B. M. t., \& Henriques, D. Y. P. (2019). The effects of awareness of the perturbation during motor adaptation on hand localization. PLoS ONE, 14(8), 1-20. https://doi.org/10.1371/journal.pone.0220884

Morehead, J. R., Qasim, S. E., Crossley, M. J., \& Ivry, R. (2015). Savings upon Re-Aiming in Visuomotor Adaptation. Journal of Neuroscience, 35(42), 14386-14396. https://doi.org/10.1523/jneurosci.1046-15.2015 


\section{Running Head: EXPLICIT AND IMPLICIT VISUOMOTOR ADAPTATION}

Neville, K. M., \& Cressman, E. K. (2018). The influence of awareness on explicit and implicit contributions to visuomotor adaptation over time. Experimental Brain Research, 236(7), 2047-2059. https://doi.org/10.1007/s00221-018-5282-7

Nisbett, R. E., \& Wilson, T. D. (1977). Telling more than we can know: Verbal reports on mental processes. Psychological Review, 84(3), 231-259. https://doi.org/10.1037/0033295X.84.3.231

Norman, E., Price, M. C., \& Duff, S. C. (2006). Fringe consciousness in sequence learning: The influence of individual differences. Consciousness and cognition, 15(4), 723-760.

Ong, N. T., Larssen, B. C., \& Hodges, N. J. (2012). In the absence of physical practice, observation and imagery do not result in updating of internal models for aiming. Experimental Brain Research, 218(1), 9-19. https://doi.org/10.1007/s00221-011-2996-1

Poh, E., Carroll, T. J., \& Taylor, J. A. (2016). Effect of coordinate frame compatibility on the transfer of implicit and explicit learning across limbs. Journal of Neurophysiology, 116(3), 1239-1249. https://doi.org/10.1152/jn.00410.2016

Redding, G. M., \& Wallace, B. (1978). Sources of "overadditivity" in prism adaptation. Perception \& Psychophysics, 24(1), 58-62.

Redding, G. M., \& Wallace, B. (1993). Adaptive Coordination and Alignment of Eye and Hand. Journal of Motor Behavior, 25(2), 75-88.

Redding, G. M., \& Wallace, B. (1994). Effects of Movement Duration and Visual Feedback on Visual and Proprioceptive Components of Prism Adaptation. Journal of Motor Behavior, 26(3), 257-266.

Redding, G. M., \& Wallace, B. (1996). Adaptive spatial alignment and strategic perceptual- motor control. Journal of Experimental Psychology: Human Perception and Performance, 22(2), 
Running Head: EXPLICIT AND IMPLICIT VISUOMOTOR ADAPTATION

379.

Redding, G. M., \& Wallace, B. (2001). Calibration and alignment are separable: Evidence from prism adaptation. Journal of Motor Behavior, 33(4), 401-412.

Redding, G. M., \& Wallace, B. (2002). Strategic Calibration and Spatial Alignment: A Model From Prism Adaptation. Journal of Motor Behavior, 34(2), 126-138.

Redding, G. M., \& Wallace, B. (2003). First-Trial Adaptation to Prism Exposure. Journal of Motor Behavior, 35(3), 229-245.

Redding, G. M., \& Wallace, B. (2006). Generalization of prism adaptation. Journal of Experimental Psychology: Human Perception and Performance, 32(4), 1006.

Reingold, E M., \& Merikle, P M. (1990). On the Infer-relatedness of meory and Measurement in the Study of Unconscious Processes. Mind \& Language, 5(1). https://doi.org/10.4135/9781412972000.n327

Shanks, D. R., \& St John, M. F. (1994). How should implicit learning be characterized? Behavioral and Brain Sciences, 17(3), 427-447. https://doi.org/10.1017/S0140525X0003538X

Simani, M. C., McGuire, L. M., \& Sabes, P. N. (2007). Visual-shift adaptation is composed of separable sensory and task-dependent effects. Journal of neurophysiology, 98(5), 2827 2841.

Smith, M. A., Ghazizadeh, A., \& Shadmehr, R. (2006). Interacting adaptive processes with different timescales underlie short-term motor learning. PLoS biology, 4(6), e179.

Taylor, J. A., \& Ivry, R. B. (2011). Flexible cognitive strategies during motor learning. PLoS Computational Biology, 7(3). https://doi.org/10.1371/journal.pcbi.1001096

Taylor, J. A., Wojaczynski, G. J., \& Ivry, R. B. (2011). Trial-by-trial analysis of intermanual transfer during visuomotor adaptation. Journal of Neurophysiology, 106(6), 3157-3172. 


\section{Running Head: EXPLICIT AND IMPLICIT VISUOMOTOR ADAPTATION}

Taylor, J. A., \& Ivry, R. B. (2014). Cerebellar and Prefrontal Cortex Contributions to Adaptation, Strategies, and Reinforcement Learning. Progress in Brain Research (1st ed., Vol. 210). Elsevier B.V. https://doi.org/10.1016/B978-0-444-63356-9.00009-1

Taylor, Krakauer, \& Ivry. (2014). Explicit and Implicit Contributions to Learning in a Sensorimotor Adaptation Task. Journal of Neuroscience, 34(8), 3023-3032. https://doi.org/10.1523/JNEUROSCI.3619-13.2014

Wang, J., \& Lei, Y. (2015). Direct-effects and after-effects of visuomotor adaptation with one arm on subsequent performance with the other arm. Journal of Neurophysiology, 114(1), 468473.

Wang, J., Joshi, M., \& Lei, Y. (2011). The extent of interlimb transfer following adaptation to a novel visuomotor condition does not depend on awareness of the condition. Journal of Neurophysiology, 106(1), 259-264.

Welch, R. B., Bridgeman, B., Anand, S., \& Browman, K. E. (1993). Alternating prism exposure causes dual adaptation and generalization to a novel displacement. Perception \& Psychophysics, 54(2), 195-204.

Werner, S., Strüder, H. K., \& Donchin, O. (2019). Intermanual transfer of visuomotor adaptation is related to awareness. PLoS ONE, 14(9), 1-19. https://doi.org/10.1371/journal.pone.0220748

Werner, S., Van Aken, B. C., Hulst, T., Frens, M. A., Van Der Geest, J. N., Strüder, H. K., \& Donchin, O. (2015). Awareness of sensorimotor adaptation to visual rotations of different size. PLoS ONE, 10(4), 1-18. https://doi.org/10.1371/journal.pone.0123321

Wolpert, D. M., \& Ghahramani, Z. (2000). Computational principles of movement neuroscience. Nature neuroscience, 3(11s), 1212.

Wolpert, D. M., \& Kawato, M. (1998). Multiple paired forward and inverse models for motor 


\section{Running Head: EXPLICIT AND IMPLICIT VISUOMOTOR ADAPTATION}

control. Neural Networks, 11(7-8), 1317-1329. https://doi.org/10.1016/S0893$\underline{6080(98) 00066-5}$

Wolpert, D. M., Ghahramani, Z., \& Jordan, M. I. (1995). An internal model for sensorimotor integration. Science, 269(5232), 1880-1882.

Zarahn, E., Weston, G. D., Liang, J., Mazzoni, P., \& Krakauer, J. W. (2008). Explaining savings for visuomotor adaptation: Linear time-invariant state-space models are not sufficient. Journal of Neurophysiology, 100(5), 2537-2548. https://doi.org/10.1152/jn.90529.2008 


\section{Université d'Ottawa}

Bureau d'éthique et d'intégrité de la recherche

\section{University of Ottawa}

Office of Research Fthics and Integrity

\section{CERTIFICAT D'APPROBATION ÉTHIQUE | CERTIFICATE OF ETHICS APPROVAL}

Numéro du dossier / Ethics File

Number Titre du projet / Project Title

Type de projet / Project Type

Statut du projet / Project Status

Date d'approbation (ii/mm/aaaa) / Approval Date (dd/mm/yyvy)

Date d'expiration (jj/mm/aaaa) / Expiry Date (dd/mm/yyyy)

\section{Équipe de recherche / Research Team}

\section{Chercheur / Researcher Affiliation}

Erin CRESSMAN

Sarveraz HEIR/NI

MOGH/DDAM

Serena GOLDLIST

Mikaela BUBNA

Zacharie CHENG.BONIN

Jean-Michel BOUCHARD

Darrin WIJEYARATNAM

Ecole des sciences de l'activité physique / School of Human Kinetics

Ecole des sciences de l'actívité physique / School of Human Kinetics

Ecole des sciences de l'activité physique / School of Human Kinetics

Ecole des sciences de l'activité physique / School of Human Kinetics

Ecole des sciences de l'activité physique / School of Human Kinetics

Ecole des sciences de l'activité physique / School of Human Kinetics

Ecole des sciences de l'activité physique / School of Human Kinetics
H-03-19-3433

Explicit and Implicit Contributions to VisuoMotor Adaptation Recherche de professeur / Professor's research project Approuvé / Approved $25 / 03 / 2019$ $24 / 03 / 2020$

\section{Role}

Chercheur Principal ? Principal Investigasor Assistant de recherche f Research Assistant Assistant de recherche / Research Assistant Assistant de recherche f Research Assistant Assistant de recherche $f$ Research Assistant Assistant de recherche f Research Assistant

Co-chercheur / Co-investigator

Conditions spéciales ou commentaires / Special conditions or comments

550 , rue Cumberland, pièce 154550 Cumberland Street, Room 154 Ottawa (Ontario) K1N 6N5 Canada Ottawa, Ontario K1N 6N5 Canada

613-562-5387 * 613-562-5338 * ethique(RuOttawa.ca / ethics@uOttawa.ca www.recherche.uottawa.ca/deontologie | www.recherche.uottawa.ca/ethics 


\section{Running Head: EXPLICIT AND IMPLICIT VISUOMOTOR ADAPTATION}

\section{Université d'Ottawa}

Bureau d'éthique et d'intégrité de la recherche

\section{University of Ottawa \\ Office of Research Ethics and Integrity}

Le Comité d'éthique de la recherche (CER) de l'Université d'Ottawa, operant conformément à l' Énoncé do politique des Trois conseils (2014) et toutes autres lois et tous règlements applicables, a examiné et approuvé la demande d'éthique du projet de recherche ci-nommé.

L'approbation est valide pour la durée indiquée plus haut et est sujette aux conditions émumérées dans la section intitulée "Conditions Spéciales ou Commentaires". Le fonmulaire o Renouvellement ou Fermeture de Projet $\$$ doit être complété quatre semaines avant la date d'échéance indiquée ci-haut afin de demander un renouvellement de cette approbation éthique ou afin de fermer le dossier.

Toutes modifications apportées au projet doivent être approuvées par le CER avant leur mise en place, gauf si le participant doit être retiré en raison d'un danger immédiat ou s'il g'agit d'un changement ayant trait à des éléments administratifs ou logistiques du projet. Les chercheurs doivent aviser le CER dans les plus brefs délais de tout changement pouvant augmenter le niveau de risque aur participants ou pouvant affecter considérablement le déroulement du projet, rapporter tout évènement impréru ou indésirable et soumettre toute nouvelle information pouvant nuire à la conduite du projet ou à la gécurité des participants.

\begin{abstract}
The University of Ottawa Research Ethics Board, which operates in accordance with the Tri-Cowncil Policy Statement (2014) and other applicable laws and regulations, has examined and approved the ethics application for the above-named research project.
\end{abstract}

Ethics approval is valid for the period indicated above and is subject to the conditions listed in the section entitled "Special Conditions or Comments". The "Renewal Project Closure" form must be completedfour weeks before the abovereferenced expiry date to request a renewal of this ethics approval or closure of the file.

Any changes made to the project nust be approved by the REB before being implemented, except when necessary to remove participants from immediate endangerment or when the modification(5) only pertain to administrative or logiatical components of the project. Investigators must also promptly alert the REB of any changes that increase the risk to participant(s), any changes that considerably affect the conduct of the project, all unanticipated and harmfil events that occur, and new information that may negatively affect the conduct of the project or the safety of the participant(s).

Risna MARCOTTE

Responsable d'éthique en recherche / Protocol Officer

Pour'For Daniel LAGAREC Président(e) du/ Chair of the Comité d'éthique de la recherche en sciences sociales et humanités / Social Sciences and Humanities Research Ethics Board

550, rue Cumberland, pièce 154550 Cumberland Street, Room 154 Ottawa (Ontario) K1N 6N5 Canada Ottawa, Ontario K1N 6N5 Canada

613-582-5387 • 613-582-5338 • ethique@uOttawa.ca / ethics@uOttawa.ca www.recherche.uottawa.ca/deontologie | wow.recherche.uottawa.ca/ethics 


\title{
Appendix B \\ Explicit and Implicit Contributions in Motor Adaptation
}

\author{
Supervisor: $\quad$ Dr. Erin Cressman, \\ Assistant Professor, \\ Montpetit Hall, Room 360 \\ School of Human Kinetics, University of Ottawa \\ Ottawa, Ontario, K1N 6N5 \\ Tel: 613-562-5800 x 4264 \\ Email: erin.cressman@uOttawa.ca
}

\section{Co-Investigator(s):}

Darrin Wijeyaratnam, Ph.D. Student

School of Human Kinetics, University of Ottawa

Jean-Michel Bouchard, M.Sc. Student

School of Human Kinetics, University of Ottawa

Sarvenaz Moghaddam, M.Sc. Student

School of Human Kinetics, University of Ottawa

Serena Goldlist, M.Sc. Student

School of Human Kinetics, University of Ottawa

Mikaela Bubna, M.Sc. Student

School of Human Kinetics, University of Ottawa

Zacharie Cheng-Boivin, B.Sc.H.K. Student

School of Human Kinetics, University of Ottawa

Funding: National Science and Engineering Research Council (NSERC) 
Running Head: EXPLICIT AND IMPLICIT VISUOMOTOR ADAPTATION

\section{Background}

Most of us perform goal directed actions - i.e. reaching for a cup of coffee - without thought or effort. It is only after something goes wrong (i.e. damage to part of the brain), that we begin to appreciate how complex a job the brain has in transforming sensory signals (e.g. visual input) into appropriate action plans. The overall goal of the research being undertaken in my laboratory is to understand how the brain transforms sensory input into motor output in the "healthy" brain. This research has important implications for people suffering from neurological disorders, as it is only after we gain a fundamental understanding of the normal mechanisms underlying goal directed action that we can begin to design effective rehabilitation programs, targeted at individuals with damage to certain areas of the brain.

\section{Purpose}

The purpose of this research is to determine how the brain combines multiple sources of sensory information so that it can plan an appropriate movement and form a coherent estimate of where the limb is in space. In particular, we are looking at the integration of visual and proprioceptive information in the brain, where proprioceptive input arises from sensory receptors inside the body that enable us to localize various parts of the body in relation to each other (e.g. proprioception is the sense that allows you to touch your index finger to your nose when your eyes are closed).

\section{Subject Profile}

To be a participant you must be a right-handed, healthy (no history of neurological, and/or motor dysfunction) adult with normal or corrected-to-normal visual function, aged between 16 and 40 years.

\section{Study Procedures}

In order to examine how visual and proprioceptive inputs are integrated in the brain you will be asked to point to targets (i.e. a visual dot) with your right/left hand as fast and as accurate as possible. You may also be asked to indicate where your hand is relative to a target (i.e. left or right of the target). In order to record your hand movement, you will hold onto a robot handle (KINARM, Kingston, ON, Canada). We do not need to do any preparation of the skin. It is important to note that this robot system only captures the position of the handle (it does not capture your image (i.e. personal features) in any form).

\section{Experimental Session}

The entire experiment will be completed in Montpetit Hall, Room 403B. Each participant will complete testing on 1 day, with each testing session lasting about an 1 hour. Upon completion of the testing session(s) the experimenter will provide additional details on the visual information displayed and the hypothesized results. 
Running Head: EXPLICIT AND IMPLICIT VISUOMOTOR ADAPTATION

\section{Risks and discomforts}

The risks involved in participating in this experiment are minimal. That is, the risks are no greater than the risks experienced in everyday life. However, you might experience slight fatigue, as you will be asked to maintain focused attention throughout the experiment and perform multiple reaching movements. In attempt to ensure that you do not become fatigued, we have scheduled 5-minute breaks approximately every 15 minutes. As well, please let us know if you require longer or more frequent periods of rest.

\section{Benefits}

Thank you for taking the time to participate in this study. While there are no direct benefits to you from participating in this study, this research has important implications for people suffering from neurological disorders. It is only after we gain a fundamental understanding of the normal mechanisms underlying sensory guided action that we can begin to design effective rehabilitation programs, targeted at individuals with damage to certain areas of the brain.

\section{Anonymity and Confidentiality}

All information and data collected are coded to maintain confidentiality. Specifically, raw data will be stored using an alphanumeric coding system so that no one will be able to identify you as your name will not appear on these files. The data will be analyzed on password protected computers that only the researchers directly involved in this study will have access to. Once analyzed the data will be kept in Room 403B, Montpetit Hall, in locked filing cabinets and only the researchers directly involved in this study will have access to your data.

No records bearing your name will leave the institution. You are encouraged to request and discuss the results of the experimental trials at any time.

The data collected in this study may be published in scientific journals, as well as in a University of Ottawa MSc or $\mathrm{PhD}$ thesis. The data will be kept for a period of 10 years post-publication and will subsequently be destroyed by the physical resources service of the University of Ottawa.

\section{Voluntary Participation}

For the entire duration of the study, it is fully understood that you may refuse to participate or withdraw from the study at any time, without question. As well, you can ask the researcher any questions about any part of the research being conducted at any time. Data of participants who withdraw from the study will be destroyed immediately. 
Running Head: EXPLICIT AND IMPLICIT VISUOMOTOR ADAPTATION

\section{INFORMED CONSENT OF PARTICIPANT}

Research involving human subject require written consent of the participants.

I, , hereby volunteer to participate as a subject in the study

entitled

"Explicit and Implicit Contributions in Motor Adaptation". I have read the information presented in the above background information and I had the opportunity to ask questions to the investigator(s). I understand that my participation in this study, or indeed any research, may involve risks that are currently unforeseen.

I recognize that there will be no direct benefit to me from my participation in this study.

I understand that if I have any questions regarding the study, I may contact Dr. Erin Cressman at 613-562-5800 ext 4264. If I have any questions or complaints with regards to the ethical conduct of this study, I may contact the Protocol Officer for Ethics in Research, University of Ottawa, Tabaret Hall, 550 Cumberland Street, Room 154, Ottawa, ON K1N 6N5, tel.: 613-5625387, email: ethics@uottawa.ca .

I have been given a copy of this Background Letter and Consent Form for me to keep.

Signature of participant: Date:

Signature of researcher: Date: 


\section{Appendix C}

Edinburgh Handedness Inventory

Your participant ID:

Please indicate with a one (1) your preference in using your left or right hand in the following tasks.

Where the preference is so strong you would never use the other hand, unless absolutely forced to, put a two (2).

If you are indifferent, put a one in each column ( $1 \mid 1)$.

Some of the activities require both hands. In these cases, the part of the task or object for which hand preference is wanted is indicated in parentheses.

\begin{tabular}{|c|c|c|}
\hline Task / Object & Left Hand & Right Hand \\
\hline \multicolumn{3}{|l|}{ 1. Writing } \\
\hline \multicolumn{3}{|l|}{ 2. Drawing } \\
\hline \multicolumn{3}{|l|}{ 3. Throwing } \\
\hline \multicolumn{3}{|l|}{ 4. Scissors } \\
\hline \multicolumn{3}{|l|}{ 5. Toothbrush } \\
\hline \multicolumn{3}{|l|}{ 6. Knife (without fork) } \\
\hline \multicolumn{3}{|l|}{ 7. Spoon } \\
\hline \multicolumn{3}{|l|}{ 8. Broom (upper hand) } \\
\hline \multicolumn{3}{|l|}{ 9. Striking a Match (match) } \\
\hline \multicolumn{3}{|l|}{ 10. Opening a Box (lid) } \\
\hline Total checks: & $\mathrm{LH}=$ & $\mathrm{RH}=$ \\
\hline Cumulative Total & \multicolumn{2}{|c|}{$\mathrm{CT}=\mathrm{LH}+\mathrm{RH}=$} \\
\hline Difference & \multicolumn{2}{|c|}{$\mathrm{D}=\mathrm{RH}-\mathrm{LH}=$} \\
\hline Result & \multicolumn{2}{|c|}{$\mathrm{R}=(\mathrm{D} / \mathrm{CT}) \times 100=$} \\
\hline $\begin{array}{l}\text { Interpretation: } \\
\text { (Left Handed: } \mathrm{R}<-40 \text { ) } \\
\text { (Ambidextrous: }-40 \leq \mathrm{R} \leq+40 \text { ) } \\
\text { (Right Handed: } \mathrm{R}>+40 \text { ) }\end{array}$ & & \\
\hline
\end{tabular}




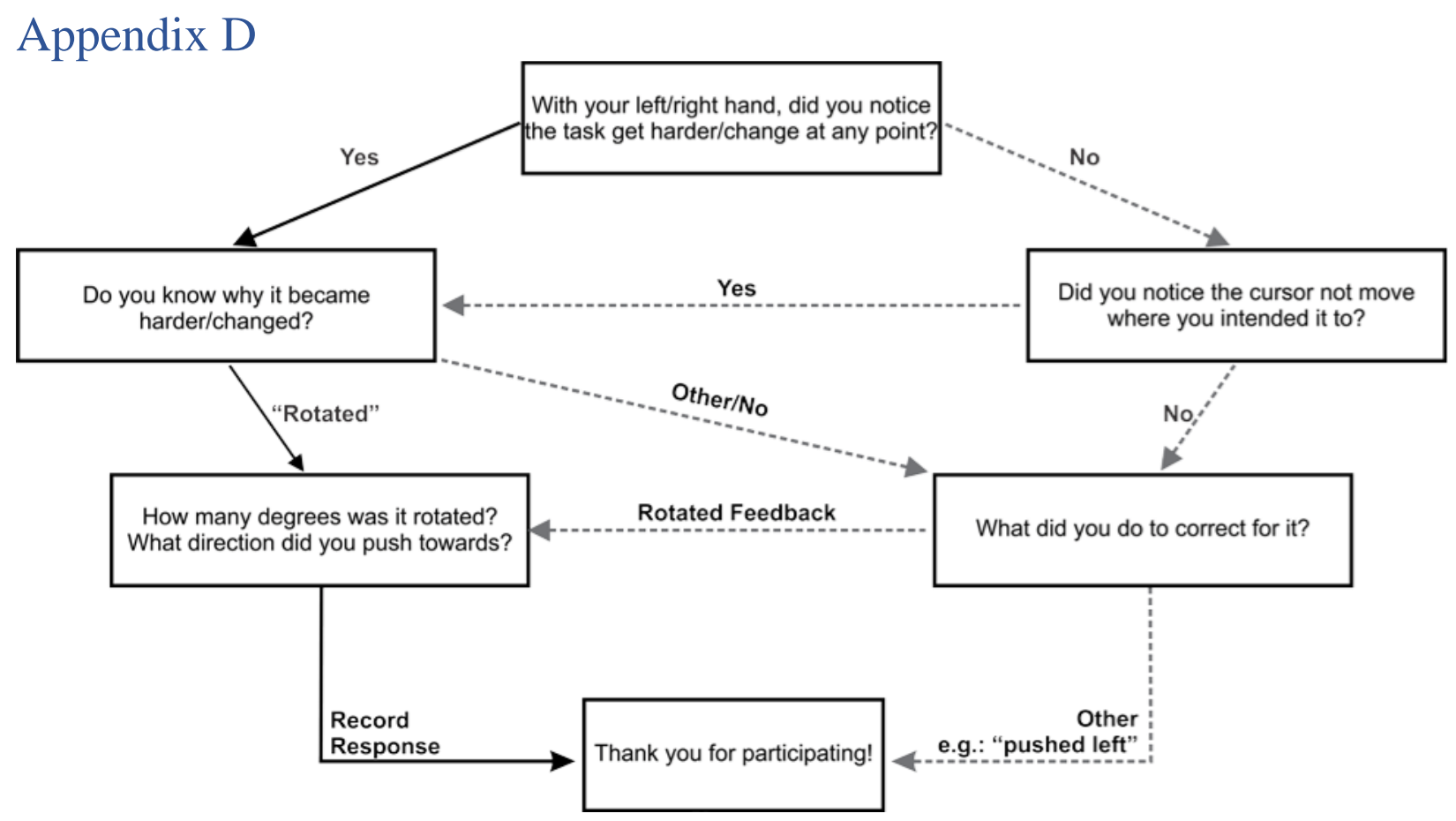

\section{Post-Experiment Questionnaire}

Questionnaire used by Benson et al. (2011) that was given to all of our participants upon completion of the study (i.e., following all reaching trials). The researcher asked a series of questions as outlined above, depending on how a participant responded. In accordance with Benson, participants were classified as being aware of the visuomotor distortion if they report using a strategy to counteract the distortion, or noticed a difference in how they had to reach in order to get the cursor to the intended target. Those reporting otherwise, were classified as unaware of the visuomotor distortion. 


\section{Appendix E}

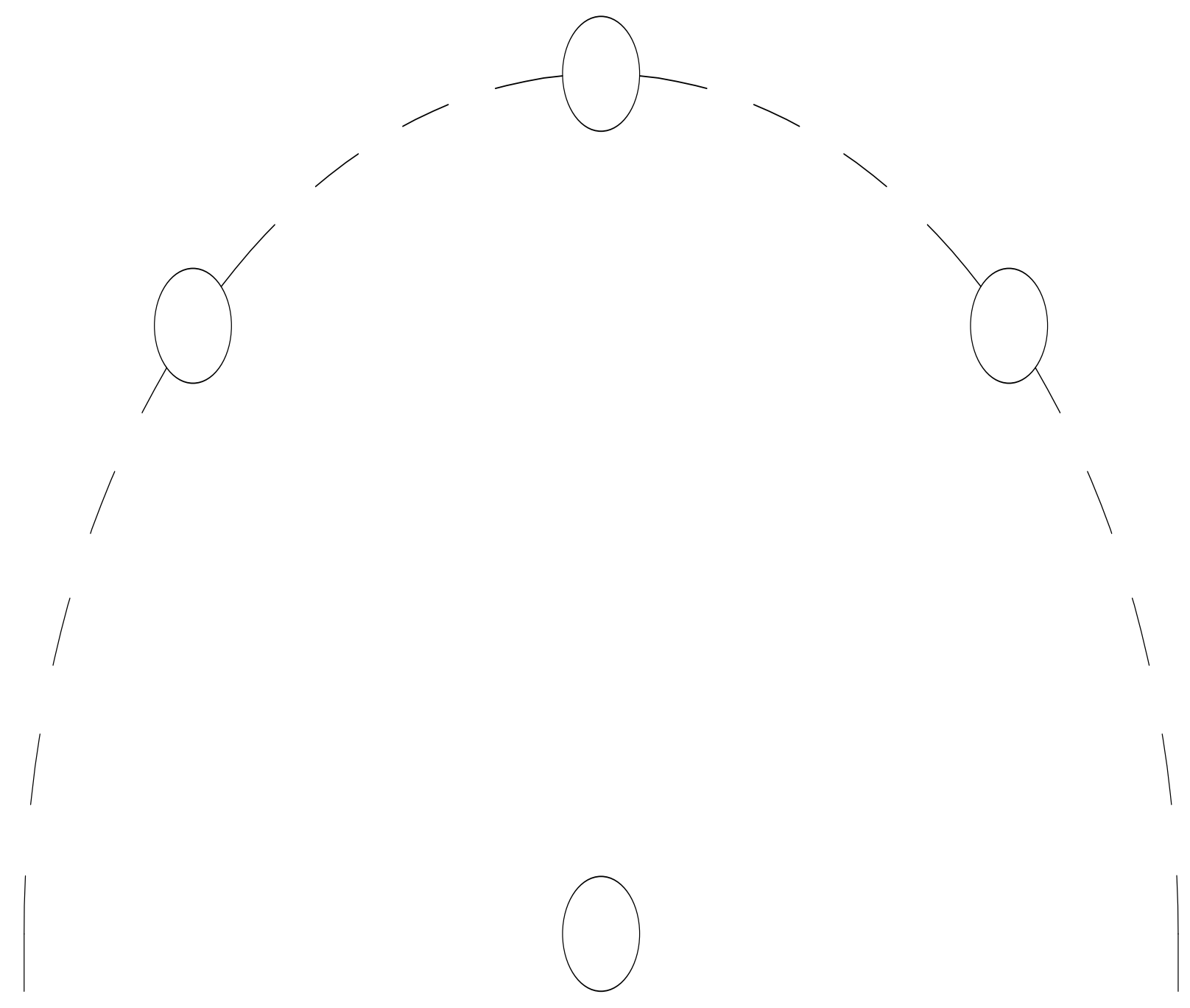

\section{Final Drawing Model}

The diagram on which participants drew where they reached in order to get the cursor to each of the targets in the rotated cursor reach training trials (i.e., reach training trials block 4). This drawing model was given to the participants at the end of all of the reach training trials, following the post-experiment questionnaire. The size of the paper was to scale to the real dimensions participants used to reach on the Kinarm. 
Running Head: EXPLICIT AND IMPLICIT VISUOMOTOR ADAPTATION

\section{Appendix F}

Participant form

Full name:

Date:

Date of Birth:

Age: Sex: M F Other

Right handed corrected vision: $\mathrm{Y} \quad \mathrm{N}$

\section{For experimenter to complete}

Position measurements

Arm Length:

Shoulder-Table Horizontal Distance:

Chair Distance:

Shoulder Table Horizontal Distance:

Eye-Table Horizontal Distance:

Shoulder - Corner Distance:

Eye-Table Vertical Distance:

Chair Height:

Eye-Corner Distance:

Testing info:

Group: PDP VRF VRF No-Cursor

Block 1: Aligned training

Block 1: No cursor Exclusion (12 trials)

BREAK

Block 1: No cursor Exclusion (12 trials)

Block 2: Rotation training

Block 2: Inclusion/Exclusion (12 trials)

\section{BREAK}

Block 2: Inclusion/Exclusion (12 trials) 
Block 3: Rotation training

Block 3: Inclusion/Exclusion (12 trials)

\section{BREAK}

Block 3: Inclusion/Exclusion (12 trials)

Block 4: Rotation training

Block 4: Inclusion/Exclusion (12 trials)

\section{BREAK}

Block 4: Inclusion/Exclusion (12 trials)

Group: PDP VRF VRF No-Cursor

Block 1: Aligned training (45 trials)

Block 1: Verbal No cursor (12 trials)

\section{BREAK}

Block 1: Verbal No cursor (12 trials)

Block 2: Rotation training (45 trials)

Block 2: Verbal No cursor (12 trials)

\section{BREAK}

Block 2: Verbal No cursor (12 trials)

Block 3: Rotation training

Block 3: Verbal No cursor (12 trials)

\section{BREAK}

Block 3: Verbal No cursor (12 trials)

Block 4: Rotation training

Block 4: Verbal No cursor (12 trials) 
Running Head: EXPLICIT AND IMPLICIT VISUOMOTOR ADAPTATION

\section{BREAK}

Block 4: Verbal No cursor (12 trials)

Group: PDP VRF VRF No-Cursor

Block 1: Aligned training (45 trials)

Block 1: Verbal cursor (12 trials)

BREAK

Block 1: Verbal cursor (12 trials)

Block 2: Rotation training (45 trials)

Block 2: Verbal cursor (12 trials)

\section{BREAK}

Block 2: Verbal cursor (12 trials)

Block 3: Rotation training

Block 3: Verbal cursor (12 trials)

BREAK

Block 3: Verbal cursor (12 trials)

Block 4: Rotation training

Block 4: Verbal cursor (12 trials)

\section{BREAK}

Block 4: Verbal cursor (12 trial) 


\section{Appendix G \\ Testing Script \\ PDP Group \\ Block 1: Introduce the baseline condition}

A) In this task, you will see a yellow target come up. Wait until it turns blue and then reach to it as quickly as possible with the goal of the cursor landing on the target. Once you are done, hold your hand still until the target disappears. After the target has disappeared, the robot will move your hand back to the home position (the white circle) to begin the next trial. Do you have any questions? Do a few practice trials - we will try a few trials now. There are 45 trials in this baseline block, and it will take about 6 minutes. Are you ready to begin?

B) You are now going to reach when you cannot see your hand - there will be no visual cursor on the screen. Once again just reach out to the target as you just did once it turns blue. The goal is to get your hand to the target. Do 6 trials, pause for a few seconds, do 6 more

C) 5-minute break

D) You are now going to reach when you cannot see your hand - there will be no visual cursor on the screen. Once again just reach out to the target as you just did once it turns blue. The goal is to get your hand to the target. Do 6 trials, pause for a few seconds, do 6 more

Block 2: Introduce the training block

A) This block is called the training block. There are 45 trials in this block, and it will take about 6 minutes. The target will come up. Once it turns blue, reach out as quickly as possible so that the cursor lands on the target. So again, you will see that cursor. Reach out so that it lands on the target.

B) You are now going to reach when you cannot see your hand - there will be no visual cursor on the screen. Once again just reach out to the target as you just did once it turns blue. However, I have two sets of instructions for you. For these first 6 trials (Exclusion trials), I want you to: "Do not use what you may have learned to get the cursor to the target. Instead, aim so that your hand goes straight to the target as you did during baseline”. For these next 6 trials (Inclusion trials): “. Use what you have learned during training in order to get the cursor to the target. In other words, aim so that the cursor would have gone straight to the target, as in the training trials you just completed"

C) 5-minute break

D) You are now going to reach when you cannot see your hand - there will be no visual cursor on the screen. Once again just reach out to the target as you just did once it turns blue. However, I have two sets of instructions for you. For these first 6 trials (Exclusion trials), I want you to: "Do not use what you may have learned to get the cursor to the target. Instead, aim so that your hand goes straight to the target as you did during baseline”. For these next 6 trials (Inclusion trials): “. Use what you have learned during training in order to get the cursor to the target. In other words, aim so that the cursor would have gone straight to the target, as in the training trials you just completed"

Block 3: Training block

A) These are like the trials you did before. This block is again called the training block. There are 45 trials in this block, and it will take about 5 minutes. The target will come up. 
Once it turns blue, reach out as quickly as possible so that the cursor lands on the target. So again, you will see that cursor. Reach out so that it lands on the target.

B) You are now going to reach when you cannot see your hand - there will be no visual cursor on the screen. Once again just reach out to the target as you just did once it turns blue. However, I have two sets of instructions for you. For these first 6 trials (Exclusion trials), I want you to: "Do not use what you may have learned to get the cursor to the target. Instead, aim so that your hand goes straight to the target as you did during baseline". For these next 6 trials (Inclusion trials): “. Use what you have learned during training in order to get the cursor to the target. In other words, aim so that the cursor would have gone straight to the target, as in the training trials you just completed"

C) 5-minute break

D) You are now going to reach when you cannot see your hand - there will be no visual cursor on the screen. Once again just reach out to the target as you just did once it turns blue. However, I have two sets of instructions for you. For these first 6 trials (Exclusion trials), I want you to: "Do not use what you may have learned to get the cursor to the target. Instead, aim so that your hand goes straight to the target as you did during baseline". For these next 6 trials (Inclusion trials): “. Use what you have learned during training in order to get the cursor to the target. In other words, aim so that the cursor would have gone straight to the target, as in the training trials you just completed"

Block 4: Last training block

A) These are like the trials you did before. This block is the last training block. There are 45 trials in this block, and it will take about 5 minutes. The target will come up. Once it turns blue, reach out as quickly as possible so that the cursor lands on the target. So again, you will see that cursor. Reach out so that it lands on the target.

B) You are now going to reach when you cannot see your hand - there will be no visual cursor on the screen. Once again just reach out to the target as you just did once it turns blue. However, I have two sets of instructions for you. For these first 6 trials (Exclusion trials), I want you to: "Do not use what you may have learned to get the cursor to the target. Instead, aim so that your hand goes straight to the target as you did during baseline". For these next 6 trials (Inclusion trials): “. Use what you have learned during training in order to get the cursor to the target. In other words, aim so that the cursor would have gone straight to the target, as in the training trials you just completed"

C) 5-minute break

D) You are now going to reach when you cannot see your hand - there will be no visual cursor on the screen. Once again just reach out to the target as you just did once it turns blue. However, I have two sets of instructions for you. For these first 6 trials (Exclusion trials), I want you to: "Do not use what you may have learned to get the cursor to the target. Instead, aim so that your hand goes straight to the target as you did during baseline". For these next 6 trials (Inclusion trials): “. Use what you have learned during training in order to get the cursor to the target. In other words, aim so that the cursor would have gone straight to the target, as in the training trials you just completed" 


\section{VRF group}

Block 1: Introduce the baseline condition

A) In this task, you will see a yellow target come up. Wait until it turns blue and then reach to it as quickly as possible with the goal of the cursor landing on the target. Once you are done, hold your hand still until the target disappears. After the target has disappeared, the robot will move your hand back to the home position (the white circle) to begin the next trial. Do you have any questions? Do a few practice trials - we will try a few trials now. There are 45 trials in this baseline block, and it will take about 6 minutes. Are you ready to begin?

B) Now - the visual display will change a bit. "You may have noticed that there are little numbers flanking the target. I would like you to tell me, before moving, the number that you think you should aim to in order to get your cursor on the target. So if you think that you should aim directly at the target, then please say " 0 ". But if you think that you should aim somewhere else, please tell me what that number is". Any questions? Ready to begin? Tell me the number of where you should aim in order to have the cursor move straight to the target. The target will then turn blue and you can reach.

C) 5-minute break

D) Now - the visual display will change a bit. "You may have noticed that there are little numbers flanking the target. I would like you to tell me, before moving, the number that you think you should aim to in order to get your cursor on the target. So if you think that you should aim directly at the target, then please say " 0 ". But if you think that you should aim somewhere else, please tell me what that number is". Any questions? Ready to begin? Tell me the number of where you should aim in order to have the cursor move straight to the target. The target will then turn blue and you can reach. After done - that was our baseline condition.

Block 2: Introduce the training block

A) This block is called the training block. There are 45 trials in this block, and it will take about 6 minutes. The target will come up. Once it turns blue, reach out as quickly as possible so that the cursor lands on the target. So again, you will see that cursor. Reach out so that it lands on the target.

B) Now - the visual display will change a bit. "You may have noticed that there are little numbers flanking the target. I would like you to tell me, before moving, the number that you think you should aim to in order to get your cursor on the target. So, if you think that you should aim directly at the target, then please say " 0 ". But if you think that you should aim somewhere else, please tell me what that number is". Any questions? Ready to begin? Tell me the number of where you should aim in order to have the cursor move straight to the target. The target will then turn blue and you can reach.

C) 5-minute break

D) Now - the visual display will change a bit. "You may have noticed that there are little numbers flanking the target. I would like you to tell me, before moving, the number that you think you should aim to in order to get your cursor on the target. So, if you think that you should aim directly at the target, then please say " 0 ". But if you think that you should aim somewhere else, please tell me what that number is". Any questions? Ready to begin? Tell me the number of where you should aim in order to have the cursor move straight to the target. The target will then turn blue and you can reach.

Block 3: Additional training block 
A) This block is called the training block. There are 45 trials in this block, and it will take about 6 minutes. The target will come up. Once it turns blue, reach out as quickly as possible so that the cursor lands on the target. So again, you will see that cursor. Reach out so that it lands on the target.

B) Now - the visual display will change a bit. "You may have noticed that there are little numbers flanking the target. I would like you to tell me, before moving, the number that you think you should aim to in order to get your cursor on the target. So, if you think that you should aim directly at the target, then please say " 0 ". But if you think that you should aim somewhere else, please tell me what that number is". Any questions? Ready to begin? Tell me the number of where you should aim in order to have the cursor move straight to the target. The target will then turn blue and you can reach.

C) 5-minute break

D) Now - the visual display will change a bit. "You may have noticed that there are little numbers flanking the target. I would like you to tell me, before moving, the number that you think you should aim to in order to get your cursor on the target. So, if you think that you should aim directly at the target, then please say " 0 ". But if you think that you should aim somewhere else, please tell me what that number is". Any questions? Ready to begin? Tell me the number of where you should aim in order to have the cursor move straight to the target. The target will then turn blue and you can reach.

Block 4: Additional training block

A) This block is called the training block. There are 45 trials in this block, and it will take about 6 minutes. The target will come up. Once it turns blue, reach out as quickly as possible so that the cursor lands on the target. So again, you will see that cursor. Reach out so that it lands on the target.

B) Now - the visual display will change a bit. "You may have noticed that there are little numbers flanking the target. I would like you to tell me, before moving, the number that you think you should aim to in order to get your cursor on the target. So, if you think that you should aim directly at the target, then please say " 0 ". But if you think that you should aim somewhere else, please tell me what that number is". Any questions? Ready to begin? Tell me the number of where you should aim in order to have the cursor move straight to the target. The target will then turn blue and you can reach.

C) 5-minute break

D) Now - the visual display will change a bit. "You may have noticed that there are little numbers flanking the target. I would like you to tell me, before moving, the number that you think you should aim to in order to get your cursor on the target. So, if you think that you should aim directly at the target, then please say " 0 ". But if you think that you should aim somewhere else, please tell me what that number is". Any questions? Ready to begin? Tell me the number of where you should aim in order to have the cursor move straight to the target. The target will then turn blue and you can reach. 


\section{VRF No-Cursor Group}

Block 1: Introduce the baseline condition

A) In this task, you will see a yellow target come up. Wait until it turns blue and then reach to it as quickly as possible with the goal of the cursor landing on the target. Once you are done, hold your hand still until the target disappears. After the target has disappeared, the robot will move your hand back to the home position (the white circle) to begin the next trial. Do you have any questions? Do a few practice trials - we will try a few trials now. There are 45 trials in this baseline block, and it will take about 6 minutes. Are you ready to begin?

B) Now - the visual display will change a bit. "You may have noticed that there are little numbers flanking the target. I would like you to tell me, before moving, the number that you think you should aim to in order to get your cursor on the target. So, if you think that you should aim directly at the target, then please say " 0 ". But if you think that you should aim somewhere else, please tell me what that number is". Ready to begin? Tell me the number of where you should aim in order to have the cursor move straight to the target. The target will then turn blue and you can reach out. You will not see the cursor when you reach. When you think you are at the target, just stop your movement. Any questions?

C) 5-minute break

D) Now - the visual display will change a bit. "You may have noticed that there are little numbers flanking the target. I would like you to tell me, before moving, the number that you think you should aim to in order to get your cursor on the target. So, if you think that you should aim directly at the target, then please say " 0 ". But if you think that you should aim somewhere else, please tell me what that number is". Ready to begin? Tell me the number of where you should aim in order to have the cursor move straight to the target. The target will then turn blue and you can reach out. You will not see the cursor when you reach. When you think you are at the target, just stop your movement. Any questions?

Block 2: Introduce the training block

A) This block is called the training block. There are 45 trials in this block, and it will take about 6 minutes. The target will come up. Once it turns blue, reach out as quickly as possible so that the cursor lands on the target. So again, you will see that cursor. Reach out so that it lands on the target.

B) Now - the visual display will change a bit. "You may have noticed that there are little numbers flanking the target. I would like you to tell me, before moving, the number that you think you should aim to in order to get your cursor on the target. So, if you think that you should aim directly at the target, then please say " 0 ". But if you think that you should aim somewhere else, please tell me what that number is". Ready to begin? Tell me the number of where you should aim in order to have the cursor move straight to the target. The target will then turn blue and you can reach out. You will not see the cursor when you reach. When you think you are at the target, just stop your movement. Any questions?

C) 5-minute break

D) Now - the visual display will change a bit. "You may have noticed that there are little numbers flanking the target. I would like you to tell me, before moving, the number that you think you should aim to in order to get your cursor on the target. So, if you think that 
you should aim directly at the target, then please say "0". But if you think that you should aim somewhere else, please tell me what that number is". Ready to begin? Tell me the number of where you should aim in order to have the cursor move straight to the target. The target will then turn blue and you can reach out. You will not see the cursor when you reach. When you think you are at the target, just stop your movement. Any questions?

Block 3: Training block

A) This block is called the training block. There are 45 trials in this block, and it will take about 6 minutes. The target will come up. Once it turns blue, reach out as quickly as possible so that the cursor lands on the target. So again, you will see that cursor. Reach out so that it lands on the target.

B) Now - the visual display will change a bit. "You may have noticed that there are little numbers flanking the target. I would like you to tell me, before moving, the number that you think you should aim to in order to get your cursor on the target. So, if you think that you should aim directly at the target, then please say "0". But if you think that you should aim somewhere else, please tell me what that number is". Ready to begin? Tell me the number of where you should aim in order to have the cursor move straight to the target. The target will then turn blue and you can reach out. You will not see the cursor when you reach. When you think you are at the target, just stop your movement. Any questions?

C) 5-minute break

D) Now - the visual display will change a bit. "You may have noticed that there are little numbers flanking the target. I would like you to tell me, before moving, the number that you think you should aim to in order to get your cursor on the target. So, if you think that you should aim directly at the target, then please say "0". But if you think that you should aim somewhere else, please tell me what that number is". Ready to begin? Tell me the number of where you should aim in order to have the cursor move straight to the target. The target will then turn blue and you can reach out. You will not see the cursor when you reach. When you think you are at the target, just stop your movement. Any questions?

Block 4: Last training block

A) This block is called the training block. There are 45 trials in this block, and it will take about 6 minutes. The target will come up. Once it turns blue, reach out as quickly as possible so that the cursor lands on the target. So again, you will see that cursor. Reach out so that it lands on the target.

B) Now - the visual display will change a bit. "You may have noticed that there are little numbers flanking the target. I would like you to tell me, before moving, the number that you think you should aim to in order to get your cursor on the target. So, if you think that you should aim directly at the target, then please say "0". But if you think that you should aim somewhere else, please tell me what that number is". Ready to begin? Tell me the number of where you should aim in order to have the cursor move straight to the target. The target will then turn blue and you can reach out. You will not see the cursor when you reach. When you think you are at the target, just stop your movement. Any questions?

C) 5-minute break 


\section{Running Head: EXPLICIT AND IMPLICIT VISUOMOTOR ADAPTATION}

D) Now - the visual display will change a bit. "You may have noticed that there are little numbers flanking the target. I would like you to tell me, before moving, the number that you think you should aim to in order to get your cursor on the target. So, if you think that you should aim directly at the target, then please say " 0 ". But if you think that you should aim somewhere else, please tell me what that number is". Ready to begin? Tell me the number of where you should aim in order to have the cursor move straight to the target. The target will then turn blue and you can reach out. You will not see the cursor when you reach. When you think you are at the target, just stop your movement. Any questions? 